\title{
Perceptions and experiences of patients living with implantable cardioverter defibrillators: a systematic review and meta-synthesis
}

\author{
Sim Leng Ooi ${ }^{1}$, Hong-Gu He ${ }^{1}$, Yanhong Dong ${ }^{2}$ and Wenru Wang ${ }^{1 *}$
}

\begin{abstract}
Background: Sudden cardiac deaths have become a growing major public health concern that affects the world. Despite the various etiologies, life-threatening cardiac arrhythmias attribute the main cause of sudden cardiac deaths. Therefore in certain groups of high-risk patients, the Implantable Cardioverter Defibrillator (ICD) has been recommended as either a secondary or primary prophylactic method of prevention.

Objective: To summarise the perceptions of ICD recipients and provide an overview of their experiences with regards to the quality of life, coping strategies, and learning needs.

Methods: A systematic search was conducted using CINAHL, MEDLINE, PsycINFO, Scopus, Cochrane Library, and Web of Science. Primary research articles published from January 2005 to January 2016 that met the inclusion criteria were selected and assessed for methodological quality.

Results: Thirty-nine articles consisting of 16 qualitative studies, 22 quantitative studies, and 1 mixed methods study were included for the meta-synthesis. Findings extracted from these studies were grouped into eight subthemes with 'living the ICD experience: a constant process of redefining oneself' emerging as an over-arching theme.

Conclusion: This review provides insight into the perspectives and experiences of ICD recipients. Current evidence highlights the need for healthcare professionals to improve future care standards and develop a patient-centric holistic program that meets the specific needs of ICD recipients. Moreover, future studies are required to address the research gaps identified and also explore the perceptions of patients living with ICD in the Asian context.
\end{abstract}

Keywords: Implantable cardioverter defibrillator, Perceptions, Experiences, Quality of life, Coping strategies, Leaning needs, Systematic review

\section{Background}

Sudden cardiac arrest describes as an abrupt state where the heart ceases to pump and causes the vital organs to be deprived of oxygen supply [1]. With a grim survival rate of less than $1 \%$ globally [2], most of these cases eventually result in unanticipated sudden cardiac deaths, generally within an hour of acute symptoms onset in people who may or may not have been diagnosed of any

\footnotetext{
* Correspondence: nurww@nus.edu.sg

${ }^{1}$ Alice Lee Centre for Nursing Studies, Yong Loo Lin School of Medicine, National University of Singapore, Level 2, Clinical Research Centre, Block MD 11,10 Medical Drive, Singapore, Singapore

Full list of author information is available at the end of the article
}

underlying pre-existing fatal cardiac conditions [3]. In fact, sudden cardiac deaths have become a growing major public health concern that affects the world $[2,3]$. Life-threatening cardiac arrhythmias attribute to the main cause of sudden cardiac deaths $[3,4]$. It is estimated that ventricular tachyarrhythmias annually account for approximately 6 million of the sudden cardiac death cases worldwide [2].

The Implantable Cardioverter Defibrillator (ICD) is a device that is surgically-inserted into patients' chest for: (1) the constant monitoring and pacing of the heart rhythm; (2) anti-tachycardia pacing (ATP) which involves delivering a series of low-energy impulses to 
revert ventricular arrhythmias; and (3) defibrillation where a strong electrical shock is delivered to restore the heartbeats again $[5,6]$. The ICD has been recommended as either a secondary prevention for survivors of prior ventricular tachycardia, ventricular fibrillation, and sudden cardiac arrest episodes or as a prophylactic primary prevention for patients with pre-existing cardiac conditions [1].

Since ICD implantation is effective in lowering the mortality rates of sudden cardiac deaths and prolonging the lifespan of patients with life-threatening cardiac conditions [7], it comes to a situation where ICD recipients will have to live with the device and their underlying chronic cardiac conditions for the rest of their lives. This systematic review aims to summarise the perceptions of ICD recipients and provide an overview of their experiences with regards to the quality of life, coping mechanisms, and learning needs. The review also hopes to identify the gaps in existing literature and healthcare practices. These findings will assist healthcare professionals in improving future care standards as well as developing a patient-centric holistic program that meets the specific needs of ICD recipients, thus improving their quality of life.

\section{Methods}

\section{Search strategy}

A systematic search was conducted in CINAHL, MEDLINE, PsycINFO, Scopus, Cochrane Library and Web of Science. Search terms including implantable cardioverter defibrillator', 'ICD', 'automatic implantable cardioverter defibrillator', 'AICD', 'implantable defibrillator', 'perception', 'perspective', 'meaning', 'belief', 'attitude', 'experience', 'quality of life', 'psychosocial', 'psychological', 'physical', 'sexual', 'spiritual', 'patient education', 'knowledge', 'coping strategies', and 'support' were used in various combinations in the search process according to the Boolean instruction and truncation notation [8]. The inclusion criteria were: (1) primary research journal articles published from January 2005 to January 2016; (2) English language publications; and (3) research that focused on the perceptions and experiences of adults living with ICD. The articles were excluded if they were: (1) editorials, commentaries, conference abstracts, opinion pieces, or review papers; and (2) focused solely on ICD technicalities, healthcare professionals, caregivers, adolescents, or children.

\section{Search outcomes and quality assessment}

The initial search across all databases yielded 31,208 articles. After removing the duplicates, the remaining 17,980 articles were screened for relevance based on the titles and abstracts. Subsequently, 535 full-text articles were retrieved, and after exclusion based on the inclusion criteria, the remaining 46 full-text articles were appraised for its quality. The Joanna Briggs Institute critical appraisal checklists [9] were used depending on the research designs to assess the methodological quality of the articles for its final inclusion in this systematic review. For the purpose of conducting a high-quality meta-synthesis, the articles were critically appraised by two independent researchers (SLO and WW). Of the 46 articles, only 39 articles met at least $60 \%$ of the appraisal checklists' criteria on both independent assessments and were included in this review. The included studies are summarised in Table 1, and Fig. 1 depicts the PRISMA flowchart documenting the search process.

\section{Data extraction and analysis}

A data extraction form was used to extract information pertaining to the research aims, research designs, sampling methods, sample characteristics, outcome measures, data collection instruments, and key findings. For the extracted qualitative data, a meta-synthesis was used to integrate findings and offer a new interpretation across the reviewed articles. Findings from the quantitative studies were synthesised and presented in a narrative manner. A thematic analysis approach [10] was adopted for this systematic review. The studies were firstly read and familiarised before identifying for implicit and explicit codes across the text. Similar occurring codes captured in the study findings were then categorised.

\section{Results}

\section{Characteristics of included studies}

Among 39 studies included, there were 16 qualitative, 22 quantitative, and 1 mixed-methods. Purposive and convenience sampling were the most common methods, and only two studies used either quota or strategic theoretical sampling $[11,12]$. With the exception of two Asian studies i.e. Japan and Hong Kong [13, 14], the majority of studies were conducted in the Western countries, more frequently in the United States [15-25], Sweden [11, 12, 26-29], Norway [30-32], and Netherlands [33-36].

All of the quantitative studies $(n=22)$ were of a descriptive correlational design, and the sample size ranged from 35 to 3067 participants, in which the largest study was conducted in collaboration with the Swedish ICD and Pacemaker Registry [29]. For qualitative studies, two studies adopted a grounded theory approach whereas the other 14 studies used phenomenological and descriptive designs. While most qualitative studies reported only themes relating to the perceptions of ICD recipients, there were two particular studies that also explored and compared the experiences of their partners or caregivers. By comparison, the mixed-method study consisted of both interpretive phenomenological and 
Table 1 Summary of included studies

\begin{tabular}{|c|c|c|c|c|c|c|}
\hline $\begin{array}{l}\text { Author (Year) } \\
\text { Country }\end{array}$ & Research Aims & Research Design & Sample Characteristics & Outcome Measures & Instruments & Key Findings \\
\hline $\begin{array}{l}\text { Bilanovic et al. (2013) } \\
\text { [37] } \\
\text { Canada }\end{array}$ & $\begin{array}{l}\text { Qualitative } \\
\text { To explore experiences } \\
\text { of phantom shocks in } \\
\text { ICD recipients } \\
\text { Quantitative } \\
\text { To describe } \\
\text { psychosocial correlates } \\
\text { of objective and } \\
\text { phantom shocks }\end{array}$ & $\begin{array}{l}\text { Mixed Methods } \\
\text { Qualitative } \\
\text { Interpretive } \\
\text { phenomenology } \\
\text { Quantitative } \\
\text { Cross-sectional } \\
\text { descriptive correlational } \\
\text { quantitative }\end{array}$ & $\begin{array}{l}\text { Purposive sampling } \\
\text { (17 participants) } \\
9 \text { ICD recipients with } \\
\text { phantom shocks (PS) } \\
\text { within the last } \\
24 \text { months } \\
\text { - all males } \\
\text { - mean age: } 65.9 \text { years } \\
8 \text { ICD recipients with } \\
\text { objective shocks (OS) } \\
\text { within the last } \\
24 \text { months } \\
\text { - all males } \\
\text { - mean age: } 63.9 \text { years }\end{array}$ & $\begin{array}{l}\text { Qualitative } \\
\text { Phantom shock } \\
\text { experiences } \\
\text { (8 participants, } 1 \text { refused } \\
\text { to complete) } \\
\text { Quantitative } \\
\text { Psychosocial } \\
\text { measurements of the } \\
\text { level of: } \\
\text { - Post-traumatic stress } \\
\text { disorder (PTSD) } \\
\text { - Depression \& anxiety } \\
\text { - Disease-specific distress } \\
\text { - Social desirability }\end{array}$ & $\begin{array}{l}\text { Qualitative } \\
\text { Semi-structured interview } \\
\text { (face-to-face) } \\
\\
\text { Quantitative } \\
\text { Instruments: } \\
\text { - PTSD Checklist - Civilian } \\
\quad \text { Version (PCL-C) } \\
\text { - Hospital Anxiety \& } \\
\text { Depression Scale (HADS) } \\
\text { - Cardiac Anxiety } \\
\text { Questionnaire (CAQ) } \\
\text { - Socially Desirable Response } \\
\text { Set (SDRS-5) }\end{array}$ & $\begin{array}{l}\text { Qualitative } \\
\text { Theme 1: Phantom shock as a somatic } \\
\text { experience } \\
\text { PS is strikingly similar to OS; Vivid } \\
\text { physical sensation of 'punch in middle } \\
\text { of breast' } \\
\text { Theme 2: Emotional impact of phantom } \\
\text { shock } \\
\text { Alarmed, confused, anxious, fear, } \\
\text { helpless; Mistrust in ICD } \\
\text { Theme 3: Searching for meaning } \\
\text { Rationalize situation, trying to account } \\
\text { for the cause of PS } \\
\text { Quantitative } \\
\text { - Both PS \& OS } \uparrow \text { trauma \& anxiety } \\
\text { - PS } \uparrow \text { psychological distress (depression, } \\
\text { PSTD) \& social desirability } \\
\text { - OS } \uparrow \text { heart-focused worry }\end{array}$ \\
\hline $\begin{array}{l}\text { Bolse et al. (2005) } \\
{[15]} \\
\text { United States }\end{array}$ & $\begin{array}{l}\text { To describe the } \\
\text { perceptions of ICD } \\
\text { recipients on their life } \\
\text { situations }\end{array}$ & $\begin{array}{l}\text { Descriptive } \\
\text { phenomenology } \\
\text { (Dahlgren \& Fallsberg's } \\
\text { approach) }\end{array}$ & $\begin{array}{l}\text { Purposive sampling } \\
\text { with maximum variation } \\
\text { sampling } \\
\text { (14 participants) } \\
\text { - } 8 \text { males, } 6 \text { females } \\
\text { - mean age: } 55.71 \text { years } \\
\text { (range: } 21-84 \text { years) } \\
\text { - average } 2 \text { years with } \\
\text { ICD } \\
\text { - } 6 \text { experienced shocks } \\
\text { within the } 1 \text { st year }\end{array}$ & $\begin{array}{l}\text { Perceptions of life } \\
\text { situations with ICD }\end{array}$ & $\begin{array}{l}\text { Semi-structured interview } \\
\text { (telephone call) }\end{array}$ & $\begin{array}{l}\text { Category 1: Trust } \\
\text { - Trust in ICD } \rightarrow \text { Security \& confidence } \\
\text { for future } \\
\text { Category 2: Adaptability } \\
\text { - Adapt to limitations in life; Obligated } \\
\text { to accept restrictions; Changing habits; } \\
\text { Resume routine } \\
\text { Category 3: Empowerment } \\
\text { - Support from family \& healthcare staff; } \\
\text { Overprotection, felt dependent }\end{array}$ \\
\hline $\begin{array}{l}\text { Carroll and Hamilton } \\
\text { (2005) [16] } \\
\text { United States }\end{array}$ & $\begin{array}{l}\text { To compare the QOL } \\
\text { in those with ICD } \\
\text { shock and those who } \\
\text { did not receive shock } \\
\text { during 1st year }\end{array}$ & $\begin{array}{l}\text { Longitudinal, } \\
\text { prospective, descriptive } \\
\text { correlational } \\
\text { quantitative }\end{array}$ & $\begin{array}{l}\text { Convenience sampling } \\
\text { (59 participants; Initially } \\
81 \text { participants }-84 \% \\
\text { retention rate) } \\
16 \text { Shock group } \\
\text { - } 13 \text { males, } 3 \text { females } \\
\text { - mean age: } 57.5 \text { years } \\
43 \text { Non-shock group } \\
\text { - } 29 \text { males, } 14 \text { females } \\
\text { - mean age: } 64.8 \text { years }\end{array}$ & $\begin{array}{l}\text { Collected at two time } \\
\text { points (at implantation \& } \\
1 \text { year after): } \\
\text { - Health status } \\
\text { - Psychological distress } \\
\text { - QOL } \\
\text { Collected at one time } \\
\text { point ( } 1 \text { year after): } \\
\text { - Fear \& concerns }\end{array}$ & $\begin{array}{l}\text { Instruments: } \\
\text { - Ferrans \& Powers QOL } \\
\text { Index } \\
\text { - Medical Outcomes Study } \\
\text { Short Form-36 (SF36) } \\
\text { - Profile of Mood States } \\
\text { (POMS) } \\
\text { - Brodsky ICD Questionnaire }\end{array}$ & $\begin{array}{l}\text { At } 1 \text { year, } \\
\text { - Shock group significantly } \downarrow \text { mental } \\
\text { health \& vitality score than non-shock } \\
\text { group } \\
\text { - Shock group } \uparrow \text { anxiety, fatigue, } \\
\text { psychological distress, \& suffering than } \\
\text { non-shock group }\end{array}$ \\
\hline $\begin{array}{l}\text { Carroll and Hamilton } \\
\text { (2008) [45] } \\
\text { United States }\end{array}$ & $\begin{array}{l}\text { To investigate the } \\
\text { changes in health status, } \\
\text { QOL and psychological } \\
\text { state following ICD } \\
\text { implantation } 4 \text { years } \\
\text { later }\end{array}$ & $\begin{array}{l}\text { Longitudinal, } \\
\text { prospective, descriptive } \\
\text { correlational } \\
\text { quantitative }\end{array}$ & $\begin{array}{l}\text { Convenience sampling } \\
\text { ( } 41 \text { participants; Initially } \\
70 \text { participants - } 59 \% \\
\text { retention rate) } \\
\text { - } 30 \text { males, } 11 \text { females } \\
\text { - mean age: } 60.4 \text { years }\end{array}$ & $\begin{array}{l}\text { Collected at six time } \\
\text { points (at implantation, } \\
6 \text { months, } 1 \text { year, } 2 \text { years, } \\
3 \text { years, } 4 \text { years later): } \\
\text { - Health status } \\
\text { - Psychological distress } \\
\text { - QOL }\end{array}$ & $\begin{array}{l}\text { Instruments: } \\
\text { - Quality of Life Index-Cardiac } \\
\text { III (CQLI-3) } \\
\text { - Medical Outcomes Study } \\
\text { Short Form-36 (SF36) } \\
\text { - Profile of Mood States } \\
\text { (POMS) }\end{array}$ & $\begin{array}{l}\text { - Mental health score improved } \\
\uparrow \text { mental health \& } \downarrow \text { psychological } \\
\text { distress by } 6 \text { months post-ICD } \\
\text { - Physical score worsened } \\
\text { Physical sub-score significant } \uparrow a t 6 \text { months } \\
\text { but } \downarrow \text { functioning at } 3 \& 4 \text { years } \\
\text { - Fewer negative moods }\end{array}$ \\
\hline
\end{tabular}


Table 1 Summary of included studies (Continued)

$\begin{array}{llll}\text { Conelius (2015) [17] } & \text { To describe the } & \text { Descriptive } & \text { Convenience sampling } \\ \text { United States } & \text { experiences of women } & \text { phenomenology } & \text { (3 participants) } \\ & \text { with ICD implantation } & \text { (Colaizzi's approach) } & \text { - all Caucasian women } \\ & & \text { - age range: } 34-50 \text { years } \\ & & \text { - average } 1 \text { year with } \\ & & \text { ICD } \\ & & \text { - none experienced } \\ & & \text { shocks }\end{array}$

$\begin{array}{lll}\text { Flanagan et al. (2010) } & \text { To explore lived } & \text { Descriptive } \\ \text { [18] } & \text { experiences of patients } & \text { phenomenology } \\ \text { United States } & \text { with } 1-2 \text { years post- } & \text { (Van Manen's } \\ & \text { ICD implantation } & \text { hermeneutic } \\ & & \begin{array}{l}\text { phenomenology } \\ \text { approach) }\end{array}\end{array}$

Purposive sampling (85 Collected at one time participants) and its relation with ICD shock-related anxiety and ICD acceptance

Cross-sectional quantitative

- 65 males, 20 females - mean age: 59.7 years

point:

-ICD shock-related anxiety

ICD acceptance

Experiences of living with ICD

nstructured interview (face-to-face) sion 1.0

- Forida Patient Acceptance

Scale (FPAS) (FSAS)

Total psychological distress score $\downarrow$ significantly

Instruments: - Physical component \& mental Chinese (Hong Kong) SF-12 component $\downarrow$ than population norm Health Survey Standard Ver- - MCS (-) correlated with shock anxiety MCS (+) correlated with patient

- Shock anxiety (-) correlated with patient acceptance

- Age $(+)$ associated with FPAS Age (-) related with FSAS

- ICD shock (yes/no) does not but shock frequency $(0,1-2, \geq 3)$ \& gender significantly different on FSAS shock anxiety but not on MCS general mental functioning

Theme 1: Security blanket: If it keeps me alive, it's worth it

Sense of security $\rightarrow \downarrow$ Worry about

medical emergencies

Theme 2: A piece of cake: I do more than before

Stable/ $\uparrow Q O L$ after post-op period; ICD implantation process was easy

Theme 3: A constant reminder: I know it's there

Constant reminder of ICD by others and self; Affect body image

Theme 4: Living on the edge: I do not want it to go off

Fear of shock in public; Uncertainty over how it feels

Theme 5: Catch 22: I'd rather not have it

Rather not have but it's medically necessary; No choice, had to adjust to ICD
Purposive sampling
(14 participants)
-8 males, 6 females
median age: 55.7 yea
(range: 21-48 years)
- 10 for secondary
prevention
-average 1-2 years with
ICD
6 experienced shocks
in 1st post-op year

Experiences of patients

Unstructured interview

1-2 years after ICD

(telephone call)

implantation
Theme 1: Appreciation versus

apprehension

Gratitude; Anxiety over uncertainty of shock

Theme 2: Maintaining structure \& routine as a way to maintain sense of self

Strong need to maintain structured

routine; Reassure family that someone is checking on them

Theme 3: Isolation \& vulnerability 


\begin{tabular}{|c|c|c|c|c|}
\hline $\begin{array}{l}\text { Flemme et al. (2005) } \\
\text { [28] } \\
\text { Sweden }\end{array}$ & $\begin{array}{l}\text { - To describe theQOL } \\
\text { and uncertainty in } \\
\text { patients with ICD } \\
\text { - To predict QOL at } \\
\text { long-term follow-up }\end{array}$ & $\begin{array}{l}\text { Longitudinal, } \\
\text { descriptive correlational } \\
\text { quantitative }\end{array}$ & $\begin{array}{l}\text { Convenience sampling } \\
(35 \text { participants; Initial } \\
56 \text { participants - } 62.5 \% \\
\text { retention rate) } \\
\text { - } 23 \text { males, } 12 \text { females } \\
\text { - mean age: } 58.7 \text { years }\end{array}$ & $\begin{array}{l}\text { Collected at four time } \\
\text { points (pre-implantation, } \\
1-10 \text { months, } 11- \\
\text { 20months, } \geq 21 \text { months } \\
\text { average } 6.9 \text { years): } \\
\text { - QOL } \\
\text { - Uncertainty }\end{array}$ \\
\hline $\begin{array}{l}\text { Flemme et al. (2012) } \\
\text { [27] } \\
\text { Sweden }\end{array}$ & $\begin{array}{l}\text { - To describe the } \\
\text { coping strategies and } \\
\text { coping effectiveness } \\
6-24 \text { months post- } \\
\text { implantation } \\
\text { - To explore the factors } \\
\text { influencing coping } \\
\text { strategies }\end{array}$ & $\begin{array}{l}\text { Cross-sectional, } \\
\text { descriptive correlational } \\
\text { quantitative, multi- } \\
\text { centred }\end{array}$ & $\begin{array}{l}\text { Purposive sampling (147 } \\
\text { participants; Initial } 164 \\
\text { participants - } 89 \% \\
\text { retention rate) } \\
\text { - } 121 \text { males, } 26 \text { females } \\
\text { - mean age: } 63 \text { years } \\
\text { - } 77 \text { for secondary } \\
\text { prevention } \\
\text { - } 38 \text { experienced shocks }\end{array}$ & $\begin{array}{l}\text { Collected at one time } \\
\text { point: } \\
\text { - Anxiety \& depression } \\
\text { - Perceived control } \\
\text { - QOL } \\
\text { - Coping strategies }\end{array}$ \\
\hline
\end{tabular}

Flemme et al. (2011) To explore the [28] Sweden saturation at 13 concerns of patients living with ICD (6-24 months) and how they handle daily their lives
Grounded theory

(16 participampling analysis)

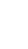
participants) - 9 males, 7 females
Purposive sampling
(16 participants; data - median age: 57.6 years (range: $31-78$ years)

-12 for secondary

prevention

- average 6-24 months with ICD

- 8 experienced shocks

Desire to connect with ICD patients but not attend support groups;

Overwhelmed by isolation from family

Theme 4: Being abandoned \& still

grieving

Resistance to accept help \& isolation $\rightarrow$ significant loss around time of illness (lost most important person); Still

grieving

Theme 5: Seeking advice, making decisions

Many unanswered queries on sexual

function \& fear shocking partner/drive

to avoid job loss/altered memory

concerns

Instruments:

Cardiac version (QLI-C)

- Overall QOL \& health/functioning

remains unchanged over time;

reasonably good at 6.9 years post $-\mathrm{ICD}$

scale Community (MUIS-C)

domains $\downarrow$ in 1st year

- Baseline to long-term follow-up, family

domain \& uncertainty

Instruments:

- Jalowiec Coping Scale-60

(JCS-60)

- Quality of Life Index -

Cardiac version (QLI-C)

- Hospital Anxiety and

Depression Scale (HADS)

- Control Attitude Scale (CAS)

Uncertainty is a predictor of low QOL

- Most seldom use coping strategies

Coping strategies used perceived as

fairly helpful

- Perceive moderate control over

condition

Optimism is the most frequently used

Optimism is the most effective coping

strategy

- Anxiety \& gender account for $26 \%$ of the variance in coping strategies

- Female use more overall, optimistic, palliative \& supportive coping

- $\uparrow$ Depression, $\uparrow$ evasive coping

$\uparrow$ Perceived control, $\downarrow$ fatalistic coping - Satisfied with QOL

Focus is not on acute Unstructured interview

phase near post- (face-to-face)

implantation.

Experiences in daily life

(had ICD for 6-24

- Concerns

- Management of

concerns
Core Category 1: Incorporating

uncertainty in daily life

Restricting activities (Strategies) Balance activity level with available resources $\rightarrow$ partly control life; Uncertain about activity level \& type to prevent shock; Fear shock $\rightarrow$ restrictions \&

careful planning of activities of daily

living (ADL)

Distracting oneself 
Accept - rec

situation (dependent on ICD \& support

from others but don't mean accept

helplessness)/body scar

Re-evaluating life

Reflective about life, changing values \& expectations; Forced to live with

uncertainty of future; Develop inner strength

\begin{tabular}{|c|c|c|c|c|c|}
\hline $\begin{array}{l}\text { Fluur et al. (2013) } \\
\text { [11] } \\
\text { Sweden }\end{array}$ & $\begin{array}{l}\text { To describe the ICD } \\
\text { recipients' experiences } \\
\text { regarding battery } \\
\text { replacement and end- } \\
\text { of-life issues }\end{array}$ & Descriptive qualitative & $\begin{array}{l}\text { Quota sampling with } \\
\text { maximum variation } \\
\text { sampling } \\
\text { ( } 37 \text { participants) } \\
\text { - } 23 \text { males, } 14 \text { females } \\
\text { - median age: } 64 \text { years } \\
\text { (range: } 29-88 \text { years) } \\
\text { - average } 4.5 \text { years with } \\
\text { ICD } \\
\text { - } 21 \text { for secondary } \\
\text { prevention } \\
\text { - } 9 \text { experienced shock } \\
\text { - } 8 \text { with ICD } \\
\text { replacement }\end{array}$ & $\begin{array}{l}\text { Experiences with battery } \\
\text { replacement \& end-of-life } \\
\text { issues }\end{array}$ & $\begin{array}{l}\text { Semi-structured interview } \\
\text { (face-to-face) }\end{array}$ \\
\hline
\end{tabular}

Theme 1: Being part of an uncertain illness trajectory

Some had insight of their condition;

some chose to ignore illness trajectory, live a day at a time

Category 1: Standing at a crossroads

Decision to replace ICD \& when to

discuss option

The unreflecting way

Replacing ICD a necessity; Offer protection from all causes of death; Adhere to doctor's decision/ICD indication

The deliberate choice

Some disagreed with doctor's advice to

not replace, unless ICD no shock $\rightarrow$

unnecessary; Some are done with life

Category 2: Progressing from one phase to another

Anticipated preferences about ICD

deactivation at end-stage

Avoiding decisions

The majority has no take on issue,

difficulty talking about death; Unaware

of deactivation option; Decide when

the time come, live each day a time

Choosing life at all costs

Most kept it as long as possible, even

with multiple shocks; Extend life;

Misunderstanding of deactivation =

immediate death/euthanasia

Facing finality

Some at end-stage reflected on mode

of death; Few will make advance deactiva-

tion planning 
Table 1 Summary of included studies (Continued)

\begin{tabular}{|c|c|c|c|c|c|c|}
\hline $\begin{array}{l}\text { Groeneveld et al. } \\
\text { (2007) [19] } \\
\text { United States }\end{array}$ & $\begin{array}{l}\text { - To measure and } \\
\text { compare the QOL } \\
\text { among primary \& } \\
\text { secondary } \\
\text { prevention } \\
\text { - To identify the } \\
\text { predictive factors for } \\
\text { QOL in each group }\end{array}$ & $\begin{array}{l}\text { Cross-sectional, } \\
\text { descriptive correlational } \\
\text { quantitative }\end{array}$ & $\begin{array}{l}\text { Purposive sampling } \\
\text { (120 participants) } \\
45 \text { Primary prevention } \\
\text { group } \\
\text { - } 28 \text { males, } 17 \text { females } \\
\text { - mean age: } 58 \text { years } \\
75 \text { Secondary } \\
\text { prevention group } \\
\text { - } 60 \text { males, } 15 \text { females } \\
\text { - mean age: } 61 \text { years }\end{array}$ & $\begin{array}{l}\text { Collected at one time } \\
\text { point: } \\
\text { - QOL } \\
\text { - ICD concerns }\end{array}$ & $\begin{array}{l}\text { Instruments: } \\
\text { - Euro-QOL-5D (EQ-5D), } \\
\text { Visual Analogue Scale (EQ- } \\
\text { VAS), } \\
\text { - Health Utilities Index-Mark } 3 \\
\text { HUI-3) } \\
\text { - Medical Outcomes } \\
\text { Questionnaires Survey Short } \\
\text { Form-12 (SF-12) } \\
\text { - Florida Patient Acceptance } \\
\text { Survey (FPAS) }\end{array}$ & $\begin{array}{l}\text { - No significant difference between } \\
\text { both groups in all QOL scales } \\
\text { - Both groups view their devices } \\
\text { favourably according to the FPAS } \\
\text { scale, no significant difference } \\
\text { - Anxiety/concerns on: } \\
\text { Lifting (40\%) } \\
\text { Sexual activity (19\%) } \\
\text { Driving (14\%) }\end{array}$ \\
\hline $\begin{array}{l}\text { Habibovic et al. } \\
\text { (2011) [33] } \\
\text { Netherlands }\end{array}$ & $\begin{array}{l}\text { To examine the effect } \\
\text { of gender versus NYHA } \\
\text { Class III/IV, ICD shock } \\
\text { and Type D personality } \\
\text { as determinantof } \\
\text { anxiety \& QOL using } \\
\text { Cohen's effect size } \\
\text { estimates }\end{array}$ & $\begin{array}{l}\text { Longitudinal, } \\
\text { descriptive correlational } \\
\text { quantitative, multi- } \\
\text { centred }\end{array}$ & $\begin{array}{l}\text { Purposive sampling } \\
\text { (718 Participants; Initial } \\
1080 \text { participants - 66\% } \\
\text { retention rate) } \\
139 \text { Female Group } \\
\text { - mean age: } 58.3 \text { years } \\
579 \text { Male Group } \\
\text { - mean age: } 61.4 \text { years }\end{array}$ & $\begin{array}{l}\text { Collected at two time } \\
\text { points (at implantation \& } \\
12 \text { months after): } \\
\text { - Anxiety } \\
\text { - QOL }\end{array}$ & $\begin{array}{l}\text { Instruments: } \\
\text { - Medical Outcomes Study } \\
\text { Short Form-36 (SF36) } \\
\text { - Spielberger's State Trait } \\
\text { Anxiety Inventory (STAI) } \\
\text { - Type D Scale (DS14) }\end{array}$ & $\begin{array}{l}\text { - No difference between men \& women } \\
\text { on mean anxiety scores } \\
\text { - QOL difference in } 2 \text { out of } 8 \text { subscales } \\
\text { of SF-36, women poorer physical } \\
\text { functioning \& vitality than men } \\
\text { - In anxiety, effect size at baseline \& } \\
12 \text { months for gender, } \\
\text { NYHA class \& ICD shocks } \rightarrow \text { small } \\
\text { Type D personality } \rightarrow \text { large } \\
\text { - In QOL, effect size at baseline \& } \\
12 \text { months, } \\
\text { Gender } \rightarrow \text { small } \\
\text { NYHA class \& Type D personality } \rightarrow \\
\text { moderate to large }\end{array}$ \\
\hline $\begin{array}{l}\text { Herman et al. (2013) } \\
\text { [50] } \\
\text { Prag }\end{array}$ & $\begin{array}{l}\text { To gain insight into } \\
\text { patients' opinions, } \\
\text { attitudes and wishes } \\
\text { regarding end-of-life } \\
\text { decisions, ICD deacti- } \\
\text { vation and their } \\
\text { knowledge }\end{array}$ & $\begin{array}{l}\text { Cross-sectional, } \\
\text { descriptive quantitative }\end{array}$ & $\begin{array}{l}\text { Convenience sampling } \\
\text { (109 participants; Initial } \\
112 \text { participants, } 3 \\
\text { excluded due to } \\
\text { incomplete } \\
\text { questionnaire) } \\
\text { - } 91 \text { males, } 18 \text { females } \\
\text { - mean age: } 67.6 \text { years } \\
\text { - average } 662.4 \text { days } \\
\text { with ICD }\end{array}$ & $\begin{array}{l}\text { Collected at one time } \\
\text { point: } \\
\text { Survey questionnaire on } \\
\text { end-of-life decisions, ICD } \\
\text { deactivation \& overall } \\
\text { understanding }\end{array}$ & $\begin{array}{l}\text { Instruments: } \\
\text { - Self-developed survey } \\
\text { questionnaire (qualitative } \\
\text { questions - yes/no; } \\
\text { quantitative questions - } \\
\text { VAS) }\end{array}$ & $\begin{array}{l}\text { - Felt safer with ICD }(90.8 \%) \\
\text { - Health status improved (60.6\%) } \\
\text { - Discussed topic with doctor }(7.3 \%) \\
\text { - Never thought of ICD deactivation at } \\
\text { end-of-life ( } 45.9 \%) \\
\text { - Wanted more information }(40.1 \%) \\
\text { - Refused additional information on the } \\
\text { issue ( } 25.7 \%) \\
41.7 \% \text { from secondary prevention \& } \\
22.4 \% \text { from primary prevention refused } \\
\text { to speak of deactivation } \\
\text { - Deactivation a personal decision, } \\
\text { won't involve relatives }(50.1 \%)\end{array}$ \\
\hline $\begin{array}{l}\text { Humphreys et al. } \\
\text { (2016) [42] } \\
\text { United Kingdom }\end{array}$ & $\begin{array}{l}\text { - To explore the } \\
\text { perceived concerns } \\
\text { and benefits of ICD } \\
\text { - To explore the } \\
\text { emotional responses } \\
\text { to ICD and coping }\end{array}$ & Descriptive qualitative & $\begin{array}{l}\text { Purposive sampling } \\
\text { (18 participants) } \\
\text { - } 11 \text { males, } 7 \text { females } \\
\text { - range } 30-68 \text { years } \\
\text { - } 5 \text { shock ( } 1 \text { out of } 5 \\
\text { female) } \\
\text { - } 13 \text { non-shock (6 out of } \\
11 \text { female) } \\
\text { - } 7 \text { for secondary } \\
\text { prevention } \\
\text { - all except } 1 \text { had }\end{array}$ & $\begin{array}{l}\text { Emotions, concerns and } \\
\text { coping of ICD recipients }\end{array}$ & $\begin{array}{l}\text { Semi-structured interview } \\
\text { (face-to-face) }\end{array}$ & $\begin{array}{l}\text { Theme 1: Physical consequences } \\
\text { Physically aware of device in body } \rightarrow \\
\text { reminds of disease; Physical } \\
\text { encumbrance - (1) Larger size (2) } \\
\text { Protrusion (3) Arm adjacent to implant } \\
\text { painful, restricted movement } \\
\text { Theme 2: Emotional consequences } \\
\text { Vulnerable/Uncertain (Non-shock } \\
\text { patients with } \\
\text { sudden cardiac arrests (SCA) episodes) }\end{array}$ \\
\hline
\end{tabular}


Table 1 Summary of included studies (Continued)

Jacq et al. (2009) [46] To assess the point France

\section{Johansson and Strömberg (2010)}

[12]

Sweden
\& QOL using

standardized scales on

shock and non-shock

patients

To describe the perceptions of ICD recipients regarding driving \& driving restrictions
Cross-sectional,

(65 participants)

40 Shock group

Collected at one time

- 35 males, 5 females

mean age: 60.18 years

average 37.44 month

with ICD

- average 7.8 shocks

25 Non-shock group

21 males, 4 females

- mean age: 59.40 years

- average 17.88 months

with ICD

Descriptive

phenomenology

(Dahlgren and

Strategic theoretical

sampling

(20 participants)

Fallsberg's approach) $\quad-14$ males, 6 females

Range: 43-82 years

-16 for secondary

prevention

- all had driving license

- 16 driving \& 4

ongoing restrictions
Traumatized; $\uparrow$ awareness of fine line between life and death; Changed

perspectives to appreciate life and work Anxiety of receiving shocks

Fear of 1st shock \& its feelings (in nonshock patients) - Male: Focus on medical implications of shocks, Female: Focus on pain \& failure to attend work Depression

Loss of confidence - (1) Inability to resume work (2) Disappoint employers $\&$ unable to support spouse $\rightarrow$ loss of status \& male role (3) $\downarrow$ financial security Loss of independence; Loss of physical fitness

Theme 3: Coping with the ICD

Avoidant/restriction; Acceptance - (1) resigned acceptance (no choice) (2)

Grateful acceptance; Set goals for 'new point:

Instruments:

- Medical Outcomes Study

Short Form-36 (SF36)

- Mini International

Neuropsychiatric Interview

according to DSM-IV (MIN

- Hospital Anxiety and

Depression Scale (HADS)

Perceptions of driving \& Unstructured interview driving restrictions

(face-to-face)
-Point prevalence of anxiety disorders in shock group

(MINI shock: 37.5\%, non-shock: $8 \%$ )

- Depressive symptoms in shock group

but point difference of depressive

disorders is insignificant

$-(+)$ correlation between the number of shocks \& depressive symptoms

$-(-)$ correlation between the number of shocks \& SF-36 mental health sub-

score

Category 1: Achieving adherence to driving restriction

Non-adherence when beliefs \&

preferences unaddressed/information unclear/given at inappropriate time Information needs

Stress pre-implantation $\rightarrow$ less receptive to information; Lack discussion of

consequences; Inconsistent

information

Individual interpretations

Interpreted restriction as

recommendation; Difficulty adapting

Driving whole life $/ 2^{\circ}$ prevention ban

$\sim 3$ months; Blame restriction rather

than condition

Willingness to adapt 
honour doctor's agreement; Anxious of

unable to do things as usua

Category 2: Emotional influence of

driving restrictions

Wanted to keep driving privileges

Loss of independence

Losses - Social life changes/

$\downarrow$ Independence/ $\downarrow$ freedom $\rightarrow$ rely on

others for ADL (felt useless/burden

others/guilt)/limited;

Changed self-image

Perceived as physically-disabled; Less

valuable; Lose personal identity; Altered

self-image (dignity \& self-respect)

Category 3: Altered views on driving

Importance of network

Family support $\rightarrow$ driven around; (+/-)

Comfort receiving help

Influence on driving behaviour

Change driving pattern - avoid driving/

partner drive/avoid heavy traffic/limit

time \& distance

Future perspectives

Anxiety of causing accident, unsuitable

driver; Unwilling to check for arrhythmia

as fear license revoked

Mert et al. (2012) [38] To describe the

Turkey
Descriptive qualitative experiences of patients using focus group with ICD
Purposive sampling (19 Living with ICD: participants)

-15 males, 4 females

- mean age: 53.5 years

- average 15.4 months

with ICD

- 13 experienced shock
Semi-structured interview

guide (focus group)

Theme 1: Experiences in the regular

activities of daily life

Restrict physical activity/quarrel/physical contact/shower alone $\rightarrow$ fear shock/ICD dislocation

Theme 2: Experiences related to social life

Cannot resume previous social activity; Cannot leave home $\rightarrow$ cellular phone

phobia; Quit/change job

Theme 3: Familial relationships

$\downarrow$ Sexual activity, partner uncomfortable; Overprotection

Theme 4: Emotional changes

Fear, nervous, anxiety (shock > no

shock), anger; Uncertainty over shock timing

Theme 5: Experiences related to ICD shocks

Prior shock symptoms; 'Blow on chest',

Anxiety, fear of death, helplessness

(multiple shocks more pain) 
Table 1 Summary of included studies (Continued)

\begin{tabular}{|c|c|}
\hline $\begin{array}{l}\text { McDonough (2009) } \\
\text { [20] } \\
\text { United States }\end{array}$ & $\begin{array}{l}\text { - To describe the } \\
\text { everyday experiences } \\
\text { of younger adults } \\
\text { (18-40 years) with } \\
\text { ICD } \\
\text { - To describe the } \\
\text { physiological and } \\
\text { psychosocial issues of } \\
\text { younger adults } \\
\text { - To identify the } \\
\text { coping strategies }\end{array}$ \\
\hline
\end{tabular}

$\begin{array}{ll}\text { Morken, et al. (2010) } & \text { To explore the } \\ \text { [30] } & \text { experience of living } \\ \text { Norway } & \text { with ICD in daily life } \\ & \text { and the long-term (a } \\ & \text { minimum of } \\ & 10 \text { months) }\end{array}$

Descriptive qualitative

Purposive sampling with maximum variation

participants)

- Young adults age 18-

40 years

14 Internet group

-6 males, 8 females

- mean age: 32.9 years ICD

- 6 experienced shock

6 Telephone group

-2 males, 4 females

- mean age: 35.2 years

- average 3.4 years with

ICD

- 3 experienced shock

Theme 6: Patients' experiences relating to receiving information/counselling from healthcare providers

Inadequate information on impacts \& shock management; Advised on driving \& conditions affecting ICD; No chance to clarify doubts; Contradictory

information received

Theme 1: A cautious transition to a new normal

Initial diagnosis: Anxiety and concern Two methods of - Physiological \& triangulation:

psychosocial impacts of - Internet group via website Anxiety; Body image concerns; Anger (written interview, email with self; Resentment towards ICD; correspondence)

with self; Reser
Depression

- Telephone group via phone Caution, awareness and security: Daily call (telephone interview) life with ICD

Cautious; Security, trust, comfort in ICD Childbearing: Passing my disease to my children

Concern of heredity cardiac conditions; Family planning - No kids/not more;

Existing children - genetic testing/ future preparations for ICD

Financial concerns

Out-of-pocket expenses; $\uparrow$ Insurance premium; ICD \& battery replacement costs; Job instability

Physiological and psychosocial

Physical restrictions; Pain, itching,

scarring $\rightarrow$ embarrassment; Shock-related pain (female > male); Fear of shock in public; Body image \& sexual concerns Strategies to manage life with an ICD: Be positive and live life to the fullest Positive; Adhere body cues; Healthy lifestyles; Online \& social support; Educate others; Future planning

Grounded theory (Strauss \& Corbin's approach)

Purposive sampling With maximum variation sampling (16 participants) mean age: 54 years (range: $25-80$ years) - average 4.5 years with ICD
Experiences of living Unstructured interview with ICD:

- Daily life

- Long-term

(face-to-face)
Core Category 1: Reconstructing the unpredictability of living with an ICD Category 1: Losing control (After shock) Uncertainty associated with the triggering of the device

No pre-physical symptoms of arrhythmia; Unpredictability $\rightarrow$ depressing; 'Struck by lightning'

Influence on the relationship with one's partner

Afraid to be alone; Dependent on partner Reduced physical activity 
Table 1 Summary of included studies (Continued)

\begin{tabular}{|c|c|}
\hline $\begin{array}{l}\text { Morken et al. (2014) } \\
\text { [31] } \\
\text { Norway }\end{array}$ & $\begin{array}{l}\text { - To investigate the } \\
\text { extent of shock } \\
\text { anxiety \& perceived } \\
\text { support from } \\
\text { healthcare } \\
\text { professionals are } \\
\text { related to PTSD } \\
\text { symptoms } \\
\text { - To examine the } \\
\text { extent of perceived } \\
\text { support from } \\
\text { healthcare } \\
\text { professionals } \\
\text { moderate } \\
\text { relationship between } \\
\text { shock anxiety \& PTSD } \\
\text { symptoms }\end{array}$ \\
\hline $\begin{array}{l}\text { Morken et al. (2014) } \\
\text { [32] } \\
\text { Norway }\end{array}$ & $\begin{array}{l}\text { To investigate the } \\
\text { extent of perceived } \\
\text { support from } \\
\text { healthcare } \\
\text { professionals and } \\
\text { shock anxiety is related } \\
\text { to device acceptance } \\
\text { among ICD recipients }\end{array}$ \\
\hline
\end{tabular}

\begin{tabular}{|c|c|}
\hline $\begin{array}{l}\text { Cross-sectional, } \\
\text { descriptive correlational } \\
\text { quantitative }\end{array}$ & $\begin{array}{l}\text { Purposive sampling } \\
\text { (167 participants) } \\
\text { - } 133 \text { males, } 34 \text { females } \\
\text { - mean age: } 64.4 \text { years } \\
\text { - } 106 \text { for secondary } \\
\text { prevention } \\
\text { - average } 57 \text { experienced } \\
\text { shocks }\end{array}$ \\
\hline $\begin{array}{l}\text { Cross-sectional, } \\
\text { descriptive correlational } \\
\text { quantitative }\end{array}$ & $\begin{array}{l}\text { Purposive sampling } \\
\text { (167 participants) } \\
\text { - } 133 \text { males, } 34 \text { females } \\
\text { - mean age: } 64.4 \text { years } \\
\text { - } 106 \text { for secondary } \\
\text { prevention } \\
\text { - average } 57 \\
\text { experienced shocks }\end{array}$ \\
\hline
\end{tabular}

Collected at one time Instruments: point:

- PTSD

- Shock anxiety

- Social support from

healthcare professionals

Revised (IES-R)

(FAS) Sock Anxiety Scale

(FSAS)

Patient Questionnaire on

Empowerment

Collected at one time point:

- ICD acceptance

- Shock anxiety

Social support from

healthcare professionals

experienced shocks

Instruments:

- Florida Patient Acceptance Scale (FPAS)

- Florida Sock Anxiety Scale (FSAS)

Patient Questionnaire on

Empowerment $\downarrow$ Physical activity to avoid shock/fea losing driving license for work $\rightarrow \downarrow$ wellbeing \& sex life; Uncertainty over

acceptable activity level; Most engage

moderate daily exercise

Category 2: Regaining control

Being normal

Resume normal life \& perceive life good

(no new shock)

Learning to trust the ICD as a life saver

Shock $\rightarrow$ remind death \& show device

functioned: Lifesaver: Grateful for new

chance

Category 3: Lacking support

Lack of continuity \& appropriate

support from healthcare professionals

Insufficient information on impacts \&

shock; Follow-up with different doctors;

Consultation time limited

Category 4: Seeking support

Managing emotions

Empathy in listening to their feelings

Seeking guidance about physical

activity

Inactive from physical discomfort

- Agree a little/strongly on constructive support (68.8\%)

Agree a little on non-constructive support (12\%)

- Experience moderate to severe PTSD symptoms (10-15\%)

- Associations between shock anxiety \& PTSD symptoms significantly moderated by perceived nonconstructive support from healthcare professionals

$\uparrow$ Non-constructive support, $\uparrow$ tendency for PTSD especially those with shock anxiety

to device acceptance
- Experience high device acceptance (84.4\%)

Experience device-related distress (4.8\%)

Constructive support from healthcare

professionals $\uparrow$ device acceptance \&

moderate (-) relationship between

shock anxiety \& device acceptance $\rightarrow$ 
Table 1 Summary of included studies (Continued)

\begin{tabular}{|c|c|c|c|c|c|c|}
\hline $\begin{array}{l}\text { Myers and James } \\
\text { (2008) [21] } \\
\text { United States }\end{array}$ & $\begin{array}{l}\text { To examine the } \\
\text { differences in ICD } \\
\text { indicators, anxiety and } \\
\text { social support } \\
\text { between ICD recipients } \\
\text { who seek support } \\
\text { group and } \\
\text { those without }\end{array}$ & $\begin{array}{l}\text { Cross-sectional, } \\
\text { descriptive comparative } \\
\text { quantitative }\end{array}$ & $\begin{array}{l}\text { Convenience sampling } \\
\text { (150 participants) } \\
73 \text { Support Attendees } \\
\text { group } \\
\text { - } 55 \text { males, } 18 \text { females } \\
\text { - mean age: } 67.71 \text { years } \\
77 \text { Support Non- } \\
\text { Attendees group } \\
\text { - } 65 \text { males, } 12 \text { females } \\
\text { - mean age: } 68.38 \text { years }\end{array}$ & $\begin{array}{l}\text { Collected at one time } \\
\text { point: } \\
\text { - Anxiety } \\
\text { - Social support \& social } \\
\text { network }\end{array}$ & $\begin{array}{l}\text { Instruments: } \\
\text { - Spielberger's State Trait } \\
\text { Anxiety Inventory (STAI) } \\
\text { - Sarason's 6-item Social Sup- } \\
\text { port Questionnaire (SSQ) }\end{array}$ & $\begin{array}{l}\text { - Support attendees higher trait anxiety } \\
\text { than non-attendees } \\
\text { Support attendees less satisfied with } \\
\text { social support than non-attendees } \\
\text { - Trait anxiety higher in those } \\
\text { diagnosed with tachycardia } \\
\uparrow \text { Satisfaction with support, } \downarrow \text { trait \& state } \\
\text { anxiety } \\
\text { - } \uparrow \text { Social network, } \downarrow \text { trait \& state anxiety } \\
\uparrow \text { Social network, } \uparrow \text { support satisfaction }\end{array}$ \\
\hline $\begin{array}{l}\text { Palacios- Ceña et al. } \\
\text { (2011) [47] } \\
\text { Spain }\end{array}$ & $\begin{array}{l}\text { To determine the } \\
\text { experience of Spanish } \\
\text { male ICD recipients }\end{array}$ & $\begin{array}{l}\text { Descriptive } \\
\text { phenomenology } \\
\text { (Giorgi approach) }\end{array}$ & $\begin{array}{l}\text { Phase 1: } \\
\text { Purposive sampling } \\
\text { Phase 2: } \\
\text { Theoretical sampling } \\
\text { (22 participants, data } \\
\text { saturation at 16) } \\
\text { - men above age } \\
18 \text { years } \\
\text { - average } 44 \text { months } \\
\text { with ICD } \\
\text { - } 17 \text { for secondary } \\
\text { prevention } \\
\text { - } 10 \text { experienced shocks }\end{array}$ & $\begin{array}{l}\text { Experiences of living } \\
\text { with ICD }\end{array}$ & $\begin{array}{l}\text { Phase 1: } \\
\text { Unstructured interview to } \\
\text { not condition or guide } \\
\text { participant } \\
\text { (face-to-face) } \\
\text { Phase } 2 \text { : } \\
\text { Semi-structured interview to } \\
\text { elicit response on specific } \\
\text { topics of interest } \\
\text { (face-to-face) } \\
\text { - Field notes } \\
-12 \text { personal letters } \\
-4 \text { diary extracts }\end{array}$ & $\begin{array}{l}\text { Theme 1: Accepting the change } \\
\text { 'Changes (improves/restricts) in mobility } \\
\text { \& loss of independence'; 'Changes in } \\
\text { family \& work status as advised to stop } \\
\text { work' - viewed (+) by senior positions/ } \\
\text { (-) by young \& lower paying jobs } \\
\text { Theme 2: Developing strategies (To } \\
\text { adapt to ICD/lllness) } \\
\text { 'Avoidance \& evasiveness' of ICD-related } \\
\text { events, avoid contact \& stay indoors; } \\
\text { 'Search for alternative information' } \\
\text { Theme 3: Rethinking their relationship } \\
\text { with their partner \& becoming } \\
\text { emotionally more distant } \\
\text { 'Importance of wife'; } \downarrow \text { 'Frequency \& } \\
\text { length of sexual relations', fear of } \\
\text { harming partner } \rightarrow \text { emotionally-distant } \\
\text { Theme 4: Giving up some of their } \\
\text { independence } \\
\text { Family support; Overprotection } \rightarrow \text { lose } \\
\text { independence but tolerated } \\
\text { Theme 5: Transformed } \\
\text { Reflection on life, changes in outlook \& } \\
\text { priorities; 'Internal change' in work, } \\
\text { relationship \& living } \\
\text { Theme 6: With life insurance } \\
\text { Love-hate attitude towards ICD } \\
\text { Theme 7: Continual uncertainty \& } \\
\text { waiting } \\
\text { 'Discharge reminds that heart is } \\
\text { deteriorating'; Waiting for discharges } \rightarrow \\
\text { uncertainty poorly-tolerated }\end{array}$ \\
\hline
\end{tabular}

prevent shock anxiety leading to poor

Non-constructive sup cceptance 
Table 1 Summary of included studies (Continued)

\begin{tabular}{|c|c|c|c|c|c|}
\hline $\begin{array}{l}\text { Palacios- Ceña et al. } \\
\text { (2011) [43] } \\
\text { Spain }\end{array}$ & $\begin{array}{l}\text { To explore the } \\
\text { experience of elderly } \\
\text { Spanish men with ICD } \\
\text { implantation }\end{array}$ & $\begin{array}{l}\text { Descriptive } \\
\text { phenomenology } \\
\text { (Giorgi's approach) }\end{array}$ & $\begin{array}{l}\text { - Purposive sampling } \\
\text { - Snowball sampling } \\
\text { (20 participants; Data } \\
\text { saturation at } 15 \\
\text { participants }+5 \\
\text { participants for } \\
\text { validation) } \\
\text { - Elderly men age } 71-83 \\
\text { years } \\
\text { - average } 52.7 \text { months } \\
\text { with ICD } \\
\text { - } 15 \text { for secondary } \\
\text { prevention } \\
\text { - } 13 \text { experienced } \\
\text { shocks/storm shocks }\end{array}$ & $\begin{array}{l}\text { Experiences of living } \\
\text { with ICD }\end{array}$ & $\begin{array}{l}\text { Unstructured interview } \\
\text { (face-to-face) } \\
\text { - Field notes } \\
\text { - } 6 \text { personal letters } \\
\text { - } 1 \text { diary }\end{array}$ \\
\hline
\end{tabular}

$\begin{array}{llll}\text { Pedersen et al. (2013) } & \text { - To examine patients' } \\ \text { [34] } & \text { knowledge and } & \begin{array}{l}\text { Cross-sectional, } \\ \text { descriptive correlational }\end{array} & \text { Convenience sampling } \\ \text { Netherlands } & \text { willingness for } & \text { quantitative } & \text { stratified into } 3 \text { groups) } \\ & \text { information } & & -110 \text { Group 1: } \\ & \text { - To identify the } & \text { De novo implanted } \\ \text { prevalence and } & -107 \text { Group 2: } \\ \text { correlates of } & \text { Moderate experience } \\ & \text { favourable attitude } & -77 \text { Group 3: } \\ & \text { towards deactivation } & \text { Considerable }\end{array}$

Collected at one time point:

- Patient's knowledg about deactivation -Wishes for information

Disorder Scale
- Patient Health
Questionnaire
- Type D Scale

Collected at one time point:

Raphael et al

(2011) [49]

To examine when end- Cross-sectional,

of-life \& desicend- Cross-sectional, Purposive sampling

vation issues should be

discussed and how

much patients wish to

know at pre-

implantation
(54 participants)

29 Group 1: No shock

group

20 males, 9 females - mean age: 71 years

ICD
When end-of-life \&

device deactivation

should be discussed

How much patients

wish to know at

pre-implantation
Instruments:

- Self-developed survey

questionnaire (qualitativ

questions - yes/no)

- Generalised Anxiety

-Type D Scale
Theme 1: Accepting changes

Limited functional capacity \& autonomy

from fear of shocks $\rightarrow$ ADL changes

Theme 2: Developing strategies to

adapt to changes arising in all areas of the recipient's life

Hide health \& ICD-related information;

Confidence in healthcare staff, never

seek other information sources; Positive

attitude

Theme 3: Living with someone

Love \& support from family; Strengthen

couple's relationship; Worry about

family \& try to stop them from being

around

Theme 4: Feel transformed

Reflection on meaning of life \& desire

to live in peace: 'Waiting' for the end

Resignation/predestination; New life

Resigna pen/pred Wasting' for the end

outlook \& priorities before it's too late
Theme 5: Live feeling safe

ICD as protector \& lifesaver; Expectation

of future shocks $\rightarrow$ uncertainty

- Most are aware ICD deactivation

option (68\%, 1/3 unaware)

- Important to inform patient of

possibility (95\%)

- Discussion of deactivation issues

个anxiety (82\%)

When should discussion take place?

(multiple responses):

Before implantation (49\%)

During the dying process (26\%)

Battery replacement (17\%)

$\downarrow$ Life expectancy (55\%)

- Made the decision for/against deactivation (246/84\%)

In favour of deactivation (195/79\%)

- Wish for a worthy death - avoidance of shocks during dying' independently associated with favourable attitude towards deactivation

Instruments: Self-developed survey Aware that ICD can be deactivated

questionnaire (qualitative \& without being explanted (38\%)

quantitative questions) - Want to be involved in deactivation decision (84\%) All willing to address end-of-life issues, none found discussion distressing

- Never considered ICD deactivation

(87\%) 
Table 1 Summary of included studies (Continued)

\begin{tabular}{|c|c|c|c|c|c|c|}
\hline & & & $\begin{array}{l}\text { - } 18 \text { for secondary } \\
\text { prevention } \\
25 \text { Group 2: Shock } \\
\text { group } \\
\text { - } 23 \text { males, } 2 \text { females } \\
\text { - mean age: } 74 \text { years } \\
\text { old } \\
\text { - average } 3.3 \text { years with } \\
\text { ICD } \\
-10 \text { for secondary } \\
\text { prevention }\end{array}$ & $\begin{array}{l}\text { Additional questions for } \\
\text { Group } 2 \text { regarding } \\
\text { deactivation \& factors } \\
\text { influencing decision }\end{array}$ & & $\begin{array}{l}\text { - When should discussion take place? } \\
\text { Prior implantation (52\%) } \\
\text { Really ill (24\%) } \\
\text { - Situations to consider deactivation: } \\
\text { Acutely unwell (82\%) } \\
\text { Frequency of shocks (70\%) } \\
\text { - Factors influencing deactivation } \\
\text { decision: } \\
\text { Prognosis }(85 \%) \\
\text { 'Quick death' }(70 \%)\end{array}$ \\
\hline $\begin{array}{l}\text { Saito et al. (2012) } \\
\text { [14] } \\
\text { Japan }\end{array}$ & $\begin{array}{l}\text { - To describe the } \\
\text { experiences of living } \\
\text { with arrhythmia \& } \\
\text { ICD } \\
\text { - To evaluate their } \\
\text { post- implantation } \\
\text { experiences } \\
\text { regarding insights on } \\
\text { obtaining appropriate } \\
\text { care for their } \\
\text { conditions }\end{array}$ & Descriptive qualitative & $\begin{array}{l}\text { No sampling method } \\
\text { specified } \\
\text { ( } 22 \text { participants) } \\
-20 \text { males, } 2 \text { females } \\
\text { - mean age: } 61.2 \text { years } \\
\text { old, (range: } 35-79 \\
\text { years) } \\
\text { - average } 14 \text { months } \\
\text { with ICD } \\
\text { - } 8 \text { experienced shocks }\end{array}$ & $\begin{array}{l}\text { Experiences of living } \\
\text { with arrhythmia \& ICD }\end{array}$ & $\begin{array}{l}\text { Semi-structured interview } \\
\text { (face-to-face) }\end{array}$ & $\begin{array}{l}\text { Category 1: Bewilderment stemming } \\
\text { from arrhythmia \& ICD implant } \\
\text { Uncertainty about one's own body } \\
\text { Uncertainty about fatal arrhythmia \& } \\
\text { necessity of ICD } \\
\text { Fear of arrhythmia ending my life } \\
\text { Anxiety related to ICD shock (without } \\
\text { shock - anxious of unknown, with } \\
\text { shock - anxious of recurrence) } \\
\text { Dissatisfaction with unforeseen results } \\
\text { of ICD } \\
\text { Dissatisfaction regarding limitations of } \\
\text { ICD \& lifestyle restraints; Discomfort of } \\
\text { having foreign object } \\
\text { Category 2: Facing reality of arrhythmia, } \\
\text { the ICD \& being able to continue life } \\
\text { Confirming \& managing lifestyle activities } \\
\text { Permissible range of safe lifestyle } \\
\text { activity; Concern on evaluating } \\
\text { expansion of lifestyle activity } \\
\text { Facing reality of the ICD \& being able to } \\
\text { continue life } \\
\text { Objectification of themselves as being } \\
\text { kept alive by machine } \\
\text { Category 3: Giving meaning to living } \\
\text { with arrhythmia \& ICD } \\
\text { Giving meaning to one's illness } \\
\text { Giving meaning to the value of ICD; } \\
\text { Coming to terms with own lifestyle, } \\
\text { acceptance } \\
\text { Recognition of one's disease } \\
\text { Objectification of disease (gaining } \\
\text { knowledge \& new outlook); Return to } \\
\text { original lifestyle despite changes in ADLs }\end{array}$ \\
\hline
\end{tabular}


Table 1 Summary of included studies (Continued)

\begin{tabular}{|c|c|c|c|c|c|c|}
\hline $\begin{array}{l}\text { Salmoirago-Blotcher } \\
\text { et al. (2012) [22] } \\
\text { United States }\end{array}$ & $\begin{array}{l}\text { To evaluate if better } \\
\text { spiritual well-being is } \\
\text { associated with lower } \\
\text { psychological distress } \\
\text { in ICD outpatients }\end{array}$ & $\begin{array}{l}\text { Cross-sectional, } \\
\text { descriptive correlational } \\
\text { quantitative }\end{array}$ & $\begin{array}{l}\text { Convenience sampling } \\
\text { (46 participants) } \\
\text { - } 32 \text { males, } 14 \text { females } \\
\text { - mean age: } 65 \text { years }\end{array}$ & $\begin{array}{l}\text { Collected at one time } \\
\text { point: } \\
\text { - Psychological distress } \\
\text { - Spiritual well-being }\end{array}$ & $\begin{array}{l}\text { Instruments: } \\
\text { - Hospital Anxiety and } \\
\text { Depression Scale (HADS) } \\
\text { - Functional Assessment of } \\
\text { Chronic Illness Therapy- } \\
\text { Spiritual Well-being (FACIT- } \\
\text { SWB) }\end{array}$ & $\begin{array}{l}\text { - } \uparrow \text { HADS, } \downarrow \text { FACIT-SWB } \\
\text { - Spiritual well-being is independently } \\
\text { associated with } \downarrow \text { psychological distress } \\
\text { in ICD outpatients } \\
\text { Spiritual well-being could be a protect- } \\
\text { ive factor against psychological distress } \\
\text { in these high-risk patients }\end{array}$ \\
\hline $\begin{array}{l}\text { Spindler et al. (2009) } \\
\text { [39] } \\
\text { Denmark }\end{array}$ & $\begin{array}{l}\text { - To examine if } \\
\text { women are at greater } \\
\text { risk of increased } \\
\text { anxiety, depression, } \\
\text { ICD concerns and } \\
\text { lower device } \\
\text { acceptance } \\
\text { - To examine if } \\
\text { women have poorer } \\
\text { QOL than men after } \\
\text { adjusted for } \\
\text { demographic and } \\
\text { clinical factors }\end{array}$ & $\begin{array}{l}\text { Cross-sectional, } \\
\text { descriptive correlational } \\
\text { quantitative }\end{array}$ & $\begin{array}{l}\text { Convenience sampling } \\
\text { ( } 535 \text { participants) } \\
97 \text { Female Group } \\
\text { - mean age: } 55.22 \text { years } \\
438 \text { Male Group } \\
\text { - mean age: } 62.94 \text { years }\end{array}$ & $\begin{array}{l}\text { Collected at one time } \\
\text { point: } \\
\text { - Anxiety \& depression } \\
\text { - QOL } \\
\text { - ICD concerns } \\
\text { - ICD acceptance }\end{array}$ & $\begin{array}{l}\text { Instruments: } \\
\text { - Hospital Anxiety and } \\
\text { Depression Scale (HADS) } \\
\text { - ICD Concerns Questionnaire } \\
\text { (ICDC) } \\
\text { - Florida Patient Acceptance4 } \\
\text { Survey (FPAS) } \\
\text { - Medical Outcomes Study } \\
\text { Short Form-36 (SF36) }\end{array}$ & $\begin{array}{l}\text { - Women } \uparrow a n x i e t y \text { than men } \\
\text { Women } \uparrow I C D \text { concerns than men } \\
\text { Differences in depression insignificant } \\
\text { - ICD patients with shocks } \uparrow a n x i e t y \\
\text { ICD patients with shocks } \uparrow I C D \text { concerns } \\
\text { - Significant gender differences for } 3 \\
\text { out of } 8 \text { subscales of SF-36 } \\
\text { Women reporting poorer HRQL on all } 3 \\
\text { subscales }\end{array}$ \\
\hline $\begin{array}{l}\text { Starrenburg et al. } \\
\text { (2014) [35] } \\
\text { Netherlands }\end{array}$ & $\begin{array}{l}\text { To examine } \\
\text { relationship between } \\
\text { gender and patient- } \\
\text { reported outcomes re- } \\
\text { garding general anx- } \\
\text { iety, device-related } \\
\text { anxiety, depression } \\
\text { and QOL }\end{array}$ & $\begin{array}{l}\text { Longitudinal descriptive } \\
\text { correlational } \\
\text { quantitative }\end{array}$ & $\begin{array}{l}\text { Purposive sampling } \\
(300 \text { participants) } \\
53 \text { Female group } \\
\text { - mean age: } 59.8 \text { years } \\
247 \text { Male group } \\
\text { - mean age: } 62.9 \text { years }\end{array}$ & $\begin{array}{l}\text { Collected at } 5 \text { time } \\
\text { points (pre-implant, } \\
\text { 2mths, } 5 \text { mths, 8mths, } \\
\text { 12mths): } \\
\text { - Anxiety \& depression } \\
\text { - Health-related quality of } \\
\text { life (HRQOL) } \\
\text { - Shock-related anxiety } \\
\text { - ICD acceptance }\end{array}$ & $\begin{array}{l}\text { Instruments: } \\
\text { - Hospital Anxiety and } \\
\text { Depression Scale (HADS) } \\
\text { - Florida Shock Anxiety Scale } \\
\text { (FSAS) } \\
\text { - Florida Patient Acceptance4 } \\
\text { Survey (FPAS) } \\
\text { - Medical Outcomes Study } \\
\text { Short Form-36 (SF-36) }\end{array}$ & $\begin{array}{l}\text { - Women has higher anxiety \& shock- } \\
\text { related anxiety than men within a year } \\
\text { - On most HRQOL subscales, no gender } \\
\text { differences except in physical } \\
\text { functioning where women reported } \\
\text { more improvement than men } \\
\text { - Gender is independently associated } \\
\text { with poorer device-related acceptance } \\
\text { - Women expressing higher levels of } \\
\text { concerns about body image than men }\end{array}$ \\
\hline $\begin{array}{l}\text { Steinke et al. (2005) } \\
\text { [23] } \\
\text { United States }\end{array}$ & $\begin{array}{l}\text { To explore the sexual } \\
\text { activity of patients \& } \\
\text { their partners post-ICD } \\
\text { implantation }\end{array}$ & $\begin{array}{l}\text { Descriptive qualitative } \\
\text { Participants recruited } \\
\text { from part of a larger } \\
\text { quantitative study } \\
\text { examining sexual issues } \\
\& \text { concerns from a } \\
\text { diverse of samples of } 2 \\
\text { support groups }\end{array}$ & $\begin{array}{l}\text { Convenience sampling } \\
\text { (12 participants) } \\
\text { ICD Patients } \\
\text { - } 10 \text { males, } 2 \text { females } \\
\text { - mean age: } 62 \text { years } \\
\text { - average } 5.3 \text { years with } \\
\text { ICD } \\
\text { - all except } 1 \text { sexually } \\
\text { active - cease all } \\
\text { sexual activity due to } \\
\text { ICD discharge } \\
\text { - } 5 \text { experienced ICD } \\
\text { discharge during } \\
\text { sexual activity } \\
\text { Partners } \\
\text { - } 1 \text { male, } 3 \text { females } \\
\text { - mean age: } 47 \text { years }\end{array}$ & $\begin{array}{l}\text { Post-ICD experiences: } \\
\text { - ICD impacts on } \\
\text { relationship \& sexual } \\
\text { relationship } \\
\text { - Effect of ICD discharges } \\
\text { on sexual activity } \\
\text { - Patient education \& } \\
\text { sexual counselling } \\
\text { needs } \\
\text { - Preferred patient } \\
\text { education } \\
\text { - Other sexual concerns }\end{array}$ & $\begin{array}{l}\text { Semi-structured interview } \\
\text { (face-to-face) }\end{array}$ & $\begin{array}{l}\text { Theme 1: Anxiety \& apprehension } \\
\text { Concerns about resuming sex } \\
\text { Partner overprotectiveness } \\
\text { Attentiveness to patients' needs } \\
\text { Fear of ICD firing with sexual activity } \\
\text { Fear \& anxiety related to } \uparrow \text { heart rate } \rightarrow \\
\text { may signal impending shock; (-) past } \\
\text { experiences; Change sexual frequency } \\
\text { Theme 2: Varying interests \& pattern of } \\
\text { sexual activity } \\
\text { Strong/ } \uparrow \text { sexual interest despite anxiety; } \\
\text { Explore other ways of affection; } \\
\text { \frequency; Backing off \& waiting } \\
\text { before resuming sex after ICD discharge } \\
\text { Theme 3: Powerfulness of ICD discharge } \\
\text { Patient - 'thunder going off chest'; } \\
\text { Partner - 'bumping together hard'; ICD } \\
\text { discharge unpredictable } \\
\text { Theme 4: A need for information \& } \\
\text { sexual counselling }\end{array}$ \\
\hline
\end{tabular}

Steinke et al. (2005) [23]

plore the sexual from part of a larger ntitative study \& concerns from a verse of samples of 2 al activity due to 
Table 1 Summary of included studies (Continued)

$\begin{array}{ll}\text { Strömberg et al. } & \text { - To describe the } \\ \text { (2014) [29] } & \text { knowledge on ICD at } \\ \text { Sweden } & \text { the end-of-life in a } \\ & \text { large national cohort } \\ & \text { of ICD recipients } \\ & \text { - To explore patient- } \\ & \text { related factors associ- } \\ & \text { ated with insufficient } \\ & \text { knowledge regarding } \\ & \text { role of ICD in end-of- } \\ & \text { life }\end{array}$
quantitative (3067 participants) - 2438 males, 629 females

- mean age: 66 years average 5 years since ICD implantation

- 1957 for secondary prevention

1056 experienced shock

Purposive sampling (11 participants)

Svanholm et al. (2015) [48] Denmark

\section{Descriptive} phenomenology Ricoeur's reflective thoughts of octogenarian with ICD/ phenomenology \& CRT-D with a battery interpretive approach) replacement due - mean age: 82.8 years (range: octogenarians 80-86 years - mean year range of implantation: 2003 - 10 for secondary prevention
Provider relationships

Preference of sharing sexual issues with healthcare staff based on knowledge level: Some staff indifferent/

uncomfortable

Educational approaches

ICD support member with knowledge \& experience; Need for information -

most prefer sexual information provided pre-discharge, reinforce advice, answer queries, individualized

Information for sexual counselling Lack of information on resuming sex

- Few scored all correct in EOL-ICD

(3\%; mean score: 6.6/11)

- Insufficient knowledge in EOL-ICD 25th percentile (29\%)

point:
- Knowledge about ethical aspects by age \& gender - impact of insufficient knowledge on tritudes

nstruments:

(EQ-5D)

$\sim 1 / 3$ thought deactivation = euthanasia Issues in Implantable Cardi- Only 1 in 10 wants deactivation during overter Defibrillator Patients terminal illness

(EOL-ICD) Questionnaire - Insufficient knowledge is associated with greater indecisiveness to make decisions on ICD deactivation in endof life or make decision that may not of-life or make decision that may not
achieve a high quality of end-of-life experience

e.g. favour replacing ICD even in seriously-ill/advanced age, keeping shock even in end-stage terminal illness

Experiences regarding: Semi-structured interview - Everyday life Theme 1: Feeling safe with the ICD The ICD: A life keeper

ICD is a necessity to prolong life; Understood ICD hinder natural death $\rightarrow$ refuse replacement The battery level is important Even with remote follow-up, appreciate going down to reassure battery level ICD shock - No problem

None had fear of shock; Some unsure if had shock - misunderstood knowledge Theme 2: The physician is an authority Being trustful

View physician role as treat actively $\rightarrow$ replace when battery low; Place lives in doctors' hands, grateful \& satisfied Feeling fine knowing nothing Surprised when told of possibility to deactivate ICD/Refuse replacement Criminal act to deactivate the ICD or refuse ICD replacement

View as an illegal act for doctors 
Table 1 Summary of included studies (Continued)

\begin{tabular}{|c|c|c|c|c|c|c|}
\hline $\begin{array}{l}\text { Thomas et al. (2009) } \\
\text { [24] } \\
\text { United States, } \\
\text { Canada \& New } \\
\text { Zealand }\end{array}$ & $\begin{array}{l}\text { - To evaluate the } \\
\text { changes in } \\
\text { depression, anxiety } \\
\text { and social support in } \\
\text { heart failure patients } \\
\text { who implanted ICD in } \\
\text { SCD-HeFT } \\
\text { - To evaluate effects of } \\
\text { ICD shocks on age } \\
\text { and NYHA class on } \\
\text { these changes }\end{array}$ & $\begin{array}{l}\text { Longitudinal, } \\
\text { descriptive correlational } \\
\text { quantitative }\end{array}$ & $\begin{array}{l}\text { Purposive sampling } \\
\text { (22 participants; Initial } \\
57 \text { participants - 38\% } \\
\text { retention rate) } \\
\text { - } 47 \text { males, } 10 \text { females } \\
\text { - all NYHA Class II/III } \\
\text { heart failure } \\
\text { - mean age: } 59.8 \text { years } \\
\text { - } 12 \text { experienced shock }\end{array}$ & $\begin{array}{l}\text { Collected at five time } \\
\text { points (Initial, 6, 12, 18, } \\
24 \text { months): } \\
\text { - Depression } \\
\text { - Anxiety } \\
\text { - Social support }\end{array}$ & $\begin{array}{l}\text { Instruments: } \\
\text { - Beck Depression Inventory- } \\
2 \text { (BDI-II) } \\
\text { - Spielberger's State Trait } \\
\text { Anxiety Inventory (STAI) } \\
\text { - Social Support } \\
\text { Questionnaire-6 (SSQ-6) }\end{array}$ & $\begin{array}{l}\text { - Depression } \downarrow \text { significantly overtime } \\
\text { overall but } \uparrow \text { in those with ICD shocks } \\
\text { - Anxiety higher in NYHA Class III than } \\
\text { Class II, } \\
\text { \in Class III but remained the same in } \\
\text { Class II } \\
\text { - Amount of social support (-) related to } \\
\text { age } \\
\text { Young, more social support } \\
\text { Social support } \downarrow \text { significantly over time } \\
\text { but young } \downarrow \text { more }\end{array}$ \\
\hline $\begin{array}{l}\text { Vazquez et al. (2008) } \\
\text { [25] } \\
\text { Australia \& United } \\
\text { States }\end{array}$ & $\begin{array}{l}\text { To investigate the } \\
\text { areas of adjustment } \\
\text { across } 3 \text { age groups of } \\
\text { women from multiple } \\
\text { centres }\end{array}$ & $\begin{array}{l}\text { Cross-sectional, } \\
\text { descriptive correlational } \\
\text { quantitative, multi- } \\
\text { centred }\end{array}$ & $\begin{array}{l}\text { Convenience sampling } \\
\text { ( } 88 \text { participants) } \\
30 \text { Young women } \\
\text { group } \\
-\leq 50 \text { years } \\
25 \text { Middle women } \\
\text { group } \\
\text { - 50-64 years } \\
32 \text { Old women group } \\
-\geq 65 \text { years } \\
\text { - average } 3.1 \text { years since } \\
\text { ICD implantation } \\
\text { - } 33 \% \text { experienced } \\
\text { shocks }\end{array}$ & $\begin{array}{l}\text { Collected at one time } \\
\text { point: } \\
\text { - Shock anxiety } \\
\text { - Death anxiety } \\
\text { - Body image concerns }\end{array}$ & $\begin{array}{l}\text { Instruments: } \\
\text { - Florida Shock Anxiety } \\
\text { Survey (FSAS) } \\
\text { - Multi-dimensional Fear of } \\
\text { Death Scale (MFODS) } \\
\text { - Florida Patient Acceptance } \\
\text { Survey (FPAS) }\end{array}$ & $\begin{array}{l}\text { - Young women has higher rate of } \\
\text { shock anxiety, death anxiety \& body } \\
\text { image concerns than middle \& older } \\
\text { women }\end{array}$ \\
\hline $\begin{array}{l}\text { Verkerk et al. (2015) } \\
\text { [36] } \\
\text { Netherlands }\end{array}$ & $\begin{array}{l}\text { - To investigate the } \\
\text { impact on QOL in 1st } \\
\text { year after ICD } \\
\text { implantation for } \\
\text { primary prevention of } \\
\text { SCD among young } \\
\text { adults between } 18 \\
\text { and } 50 \text { years } \\
\text { - To compare the QOL } \\
\text { scores with available } \\
\text { population norms }\end{array}$ & $\begin{array}{l}\text { Longitudinal, } \\
\text { descriptive quantitative }\end{array}$ & $\begin{array}{l}\text { Convenience sampling } \\
\text { ( } 35 \text { participants) } \\
-18 \text { males, } 17 \text { females } \\
\text { mean age: } 36.7 \text { years }\end{array}$ & $\begin{array}{l}\text { Collected at four time } \\
\text { points (pre-implantation, } \\
\text { 2, 6, } 12 \text { months): } \\
\text { - Depression } \\
\text { - Anxiety } \\
\text { - QOL }\end{array}$ & $\begin{array}{l}\text { Instruments: } \\
\text { - Centre for Epidemiologic } \\
\text { Studies Depression Scale } \\
\text { (CED-D) } \\
\text { - Spielberger's State Trait } \\
\text { Anxiety Inventory (STAI) } \\
\text { - Medical Outcomes Study } \\
\text { Short Form-36 (SF36) } \\
\text { - Self-designed questionnaire } \\
\text { to explore impacts of } \\
\text { receiving ICD }\end{array}$ & $\begin{array}{l}\text { - } 29 \% \text { of patients' pre-ICD depression } \\
\text { score (CES-D) higher than cut-off score } \\
\text { of } 16 \text {. } \\
\text { After } 2,6 \& 12 \text { months } \rightarrow 23,9 \& 13 \% \\
\text { respectively } \\
\text { - } 71 \% \text { of patients pre-ICD anxiety score } \\
\text { (STAI) higher than cut-off of } 40 \\
\text { After } 2,6 \& 12 \text { months } \rightarrow 40,32 \text { \& 34\% } \\
\text { respectively } \\
\text { - QOL significantly } \downarrow \text { at pre-implantation } \\
\& 2 \text { months but improved with time \& } \\
\text { is comparable with population norms } \\
\text { at } 6 \text { \& } 12 \text { months } \\
\text { - Self-designed questionnaire } 1: \text { ICD... } \\
\text { Feel protected against cardiogenic } \\
\text { condition ( } 87 \% \text { ) } \\
\text { More negative than positive effects } \\
(11 \%) \\
\text { Worry of ICD firing when nobody is } \\
\text { around ( } 22 \%) \\
\text { Influences the way I dress (16\%) } \\
\text { Can no longer do the things I enjoy } \\
\text { (19\%) } \\
\text { Lead a normal life like everyone else } \\
\text { (52\%) }\end{array}$ \\
\hline
\end{tabular}




$\begin{array}{ll}\begin{array}{l}\text { Versteeg et al. (2010) } \\ \text { [40] }\end{array} & \text { To examine if female } \\ \text { Germany } & \text { ICD patients report } \\ & \text { more psychological } \\ & \text { distress than male } \\ & \text { patients } \\ & \text { - To examine if somato- } \\ & \text { sensory amplification } \\ & \text { mediates this } \\ & \text { relationship }\end{array}$

Williams et al. (2007) To explore the

[44] experiences, concerns

Australia \& needs of ICD

recipients and their

caregivers
Cross-sectional,

descriptive correlational
quantitative

Descriptive qualitative

Purposive sampling

(22 participants)

Age range: $30-80$ years

Experiences, concerns \& Semi-structured interview

needs of recipients and (face-to-face)

11 ICD recipients

- 8 males, 3 females

- number of years with

2 years, 5 had $2-3$

years, 2 had more

than 3 years

11 Caregivers
- Self-designed questionnaire 2:

Cardiogenic condition \& ICD therapy have...

Negative influence on my professional career (34\%)

Important influence on decision for children (36\%)

- Of 29 patients with job at baseline: $28 \%$ had lost/changed their from their condition/ICD

$17 \%$ temporarily can't work

$31 \% \downarrow$ working hours

- Female has more anxiety, phobic anxiety, \& somatic health complaints than men

Female has higher somatosensory

amplification score than men - Somatosensory amplification is associated with more anxiety, phobic Somatosensory amplification mediated the association between gender \& three domains of psychological distress

Theme 1: Physical \& psychological adjustments stage

Physical difficulties; Psychological distress; Coping with reality of illness, uncertainty \& insecurity of future denial, avoidance of topic, \& refusal to resume normal activities

Theme 2: Acceptance stage - Getting on with life

- ICD accepted, normal routine resume; Strong will power

- Play it down to people/avoid discussion

- Forget about ICD being there

- Reframe interpretation of personal situation, others less fortunate; ICD support group

- Reassess lifestyle, make changes 


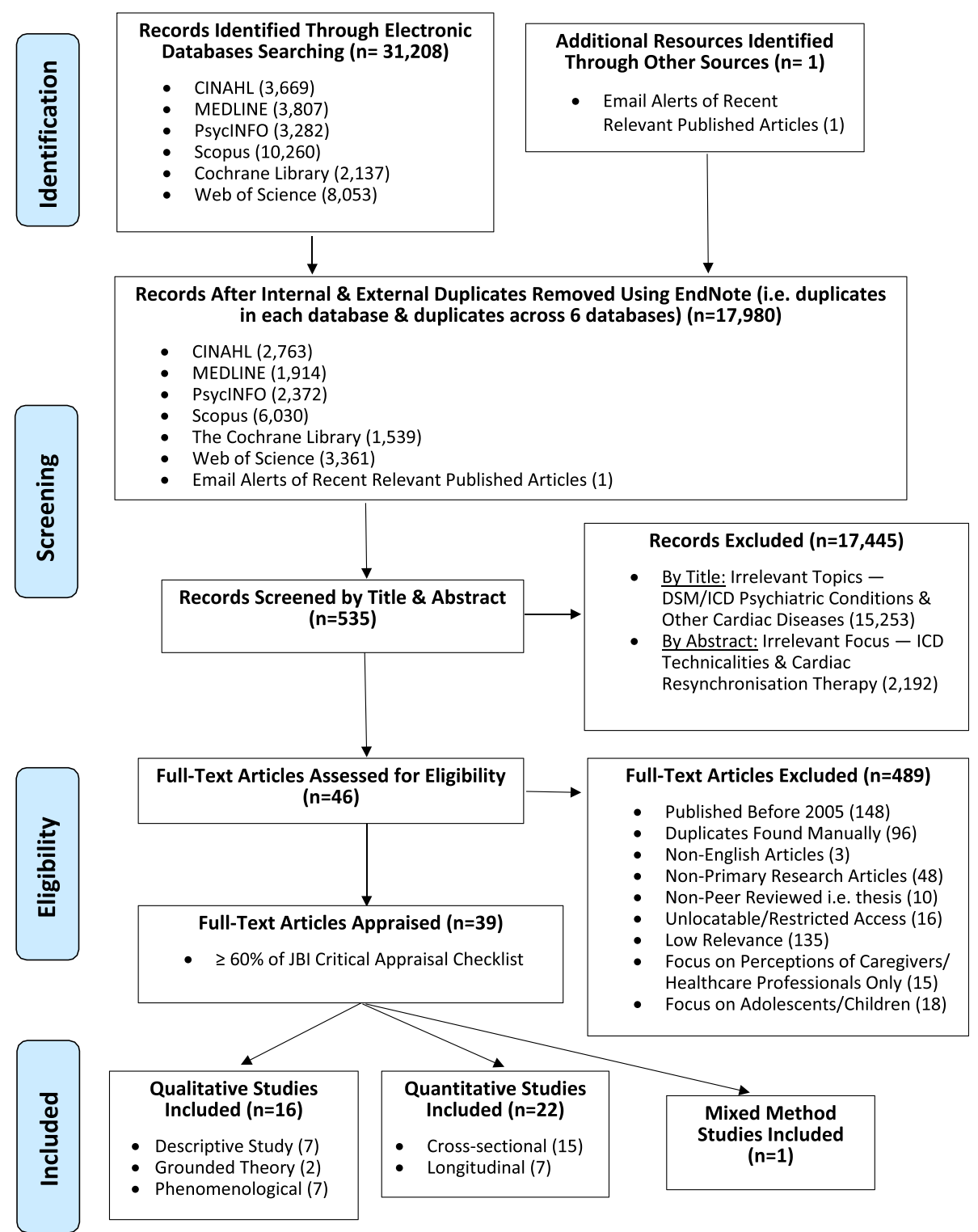

Fig. 1 PRISMA Flowchart

cross-sectional descriptive designs for a holistic understanding of the phantom shock experiences.

\section{Meta- synthesis of study findings}

Findings extracted from the studies were grouped into eight subthemes, with the synthesised finding of living the ICD experience: a constant process of redefining oneself' emerging as an over-arching theme (Table 2).

\section{Describing ICD shocks}

The shock episodes experienced by participants can be classified into: (1) objective shocks, which refer to the actual shock therapies that were delivered and recorded by the ICD; and (2) phantom shocks, the phenomena where participants reported that sensations of shock were found to be unrecorded during ICD interrogations [37]. Comparing the participants' accounts across several studies, both objective and phantom shock occurrences were found to be often abrupt and unexpected [23, 30, 37, 38]. This is because phantom shocks were predominantly encountered during sleep or sleep-wake transitions with rarer instances while awake [37]. By comparison, although some participants recalled experiencing physical symptoms of nausea, warmth, dizziness, and altered heart rhythm preceding objective shocks [20,38], the majority were unable to foresee the impending shocks [38].

Consistent across several qualitative studies, participants used terms of high intensity to describe their 
Table 2 Synthesised finding

\begin{tabular}{ll}
\hline Synthesised finding Categories & \\
\hline Living the ICD Experience: & Describing ICD Shocks \\
A Constant Process of &
\end{tabular}

A Constant Process of

Redefining Oneself
Findings
(Themes captured in the qualitative \& quantitative study)

Qualitative Study

Phantom shock as a somatic experience

Experiences related to ICD shocks

Powerfulness of ICD discharge

A cautious transition to a new normal - Physiological and psychosocial

Reconstructing the unpredictability of living with an ICD - Losing control (Uncertainty associated with the of the device)

Experiencing Uncertainty \& Psychological Distress

Qualitative Study

Emotional impact of phantom shock

Living on the edge: I do not want it to go off

Appreciation versus apprehension

Emotional influence of driving restrictions - Loss of independence

Emotional changes

A cautious transition to a new normal - Initial diagnosis: Anxiety and concern \& physiological and psychosocial

Reconstructing the unpredictability of living with an ICD - Losing control (Uncertainty associated with the triggering of the device)

Living with an ICD is living while... continual uncertainty and waiting

Bewilderment stemming from arrhythmia and ICD implant - Uncertainty about one's own body \& fear of arrhythmia ending my life

Anxiety \& apprehension - Fear of ICD firing with sexual activity

Emotional consequences - Vulnerable/uncertain, anxiety, depression

Quantitative Study

PTSD, anxiety, depression, social desirability [37]

QOL mental health score, mood states [45]

QOL, mood states, ICD concerns for shock versus non-shock [16]

QOL mental component, shock anxiety, and ICD acceptance [13]

QOL, anxiety for men versus women [33]

QOL, uncertainty [26]

QOL, ICD concerns [39]

QOL, anxiety, depression [46]

QOL mental health subscale, anxiety, depression for men versus women [39]

QOL, anxiety, body image [35]

Depression, anxiety for shock versus non-shock [24]

Shock anxiety, death anxiety, body image for young versus old women [25]

$\mathrm{QOL}$, depression, anxiety, impacts of ICD [36]

Anxiety, somatosensory amplification for men versus women [40]

Impacting Self-Identity, Self-Image \& Self-Perception

A constant reminder: I know it's there

Seeking advice, making decisions

Emotional influence of driving restrictions - Loss of independence \& changed self-image

Emotional changes

A cautious transition to a new normal - Initial diagnosis: Anxiety and concern \& physiological and psychosocial 
Table 2 Synthesised finding (Continued)

Bewilderment stemming from arrhythmia and ICD implant - Dissatisfaction with unforeseen results of ICD

Needing Support \& Maintaining Relationships

Identifying Learning Needs

Developing Coping Strategies
Qualitative Study

Empowerment - Receiving emotional and tangible layman support \& informational and tangible professional support

Isolation and vulnerability

Being abandoned and still grieving

Altered views on driving - Importance of network

Experiences related to social life

Familial relationships

Reconstructing the unpredictability of living with an ICD - Losing control (Influence on the relationship with one's partner)

Reconstructing the unpredictability of living with an ICD - Lacking support (Lack of continuity and appropriate support from healthcare professionals)

Reconstructing the unpredictability of living with an ICD - Seeking support (Managing emotions \& seeking guidance about physical activity)

Living with an ICD is living whilst... rethinking their relationship with their partner and becoming emotionally more distant

Living with an ICD is living while... giving up some of their independence

Living with someone

Anxiety and apprehension - Partner overprotectiveness

Quantitative Study

ICD acceptance, shock anxiety, professional support [31]

PTSD, shock anxiety, professional support [32]

Anxiety, social support [21]

Social support [24]

Qualitative Study

Empowerment - Informational and tangible professional support

Seeking advice, making decisions

Achieving adherence to driving restrictions - Information needs

Patients' experiences relating to receiving information/counselling from healthcare providers

A need for information and sexual counselling - Provider relationships, Educational approaches \& information for sexual counselling

Qualitative Study

Searching for meaning

Incorporating uncertainty in daily life - Distracting oneself \& re-evaluating life

A cautious transition to a new normal - Strategies to manage life with an ICD: Be positive and live life to the fullest

Living with an ICD is living while... developing strategies

Living with an ICD is living while... transformed

Developing strategies to adapt to changes arising in all areas of the recipient's life Bewilderment stemming from arrhythmia and ICD implant - Dissatisfaction with unforeseen results of ICD

Feel transformed

Giving meaning to living with arrhythmia \& ICD - Giving meaning to one's illness $\&$ recognition of one's disease

Getting on with life - Positive interpreting

Coping with the ICD 
Table 2 Synthesised finding (Continued)

Quantitative Study

$\mathrm{QOL}$, anxiety, depression, coping strategies [28]

Spiritual well-being, anxiety, depression [22]

Making Adjustments \& Gaining

Qualitative Study

Acceptance

Adaptability - Handling restlessness, tackling restrictions, \& managing daily living

A piece of cake: I do more than before

Catch 22: I'd rather not have it

Maintaining structure \& routine as a way to maintain sense of self

Incorporating uncertainty in daily life - Restricting activities

Incorporating uncertainty in daily life - Accepting being an ICD recipient

Achieving adherence to driving restrictions - Individual interpretations

\& Willingness to adapt

Altered views on driving - Influence on driving behaviour \& Future perspectives

Experiences in the regular activities of daily life

A cautious transition to a new normal - Caution, awareness and security: Daily life with an ICD

A cautious transition to a new normal - Childbearing: Passing my disease to my children \& financial concerns

Reconstructing the unpredictability of living with an ICD - Losing control (Reduced physical activity)

Reconstructing the unpredictability of living with an ICD - Regaining control (Being normal)

Living with an ICD is living while... accepting the change

Accepting changes

Facing reality of arrhythmia, the ICD, and being able to continue life Confirming and managing lifestyle activities \& facing reality of the ICD and being able to continue life

Varying interests and pattern of sexual activity

Getting on with life Lifestyle changes - Resuming normal activities, not thinking about ICD, lifestyle changes \& risk taking

Physical consequences

Quantitative Study

QOL physical health score [45]

QOL physical component [13]

QOL physical functioning for men versus women [33]

QOL physical and social functioning subscale, anxiety, depression for men versus women [39]

QOL physical functioning [35]

Planning for the End

Qualitative Study

Being part of an uncertain illness trajectory - Standing at a crossroads $\&$ progressing from one phase to another

The physician is an authority - Feeling fine knowing nothing \& criminal act to deactivate the ICD or refuse ICD replacement

Quantitative Study

ICD deactivation $[34,49,50]$

ICD deactivation knowledge [29] 
physical and sensory experiences with objective shocks. Common terms consisting of 'explosion', 'blow', 'bomb', 'shot by gun' [20, 23, 30], or terms with close associations like 'thunder' [23], 'lightning' [30, 38], and even phrases of similar meanings like 'electric shock' [38] and 'sticking your finger in the light socket' [23] illustrated the suddenness, striking, and high impact nature of objective shocks. Partners in close body contact with the participants also reported feeling a sudden repulsive force of being 'kicked' or 'thrown' which corroborated with the participants' account of experiencing objective shocks [23]. Accompanying these shocks, seeing light flashes $[23,38]$ were more commonly reported compared to hearing popping noises [23]. Participants with experiences of both objective and phantom shocks had described their intensity and characteristics to be vividly similar and indistinguishable [37]. However, upon closer examination, it was observed that the participants tend to use terms of comparatively lower intensity like 'punch' and 'kick' in their reference to phantom shocks [37].

Nevertheless, objective and phantom shocks were similar in that both physical sensations were felt mostly in the chest $[23,37,38]$ and pain was also recounted in the aftermath [23, 37, 38]. Specifically in objective shocks, pain experiences varied widely. With the majority reporting mild discomfort [20] to those experiencing multiple shocks having greater pain [38] and females describing more intense pain reaction than males [20]. In several studies, it was found that females tend to have greater anxiety than males $[35,39,40]$ and anxiety could have potentially exaggerated their pain experience as explained by the nocebo hyperalgesia phenomenon [41] Post-shock symptoms like nausea and dizziness were also reported in objective shocks $[15,20]$.

\section{Experiencing uncertainty and psychological distress}

In the initial post-ICD implantation period, participants experienced feelings of anxiety, fear, depression, helplessness, anger, insecurity, and uncertainty $[14,17,18,20,23,30,38,42-44]$. These negative emotions described in the qualitative interviews concurred with quantitative findings on poorer psychological wellbeing in the early phase $[24,28,36,45]$. Among them, fear and anxiety were the most prevalent emotions following post-discharge [20].

The majority were anxious over the unpredictability and occurrence of shocks as well as the potential loss of independence with ICD $[14,18,20,30,38,42]$. There were four quantitative studies that explored different anxiety levels between genders. Despite the differences in geographical locations and anxiety instruments, three studies reported higher anxiety levels in females than males [35, 39, 40]. Versteeg et al. [40] first established that somatosensory amplification could have mediated the association between gender and anxiety in ICD recipients. This may explain the findings since females were found to have a significantly higher somatosensory amplification than males [40]. However, Habibovic et al. [33] reported insignificant differences in anxiety levels between females and males due to the mediation effect of Type D personality.

The participants were also fearful of fatal arrhythmic deaths, shock encounters in public due to embarrassment and uncertainty of available support [14, 17, 20, 26, 44], exposure to electromagnetic interference [14, 17, 20, 23, 38], 'cellular phone phobia' [38, 44], ICD recalls [20], as well as driving restrictions if arrhythmias or shocks were detected [12]. There was also apprehension over resuming sexual activity as the majority feared of shocks hurting their partners $[18,20,23,43]$. Few studies reported on the sexual concerns associated with ICD, possibly because the participants were uncomfortable in bringing up such sensitive topics with the researchers. Moreover, some became depressed over the unpredictability of their cardiac arrhythmias [30,44] while others felt helpless over the loss of control in their lives [20,30,38]. Anger with one's limitations and resentment towards ICD [15, 20, 38] were also observed. Many still harboured insecurities over the device failing or battery depleting $[20,38,44]$ as well as the uncertainties that accompany arrhythmias [14] or awaiting ICD discharges [43].

Consistent across both qualitative and quantitative findings, participants with objective shocks reported more psychological distress and ICD concerns than their non-shock counterparts [16, 20,38, 46]. Besides being reminded of their deteriorating cardiac conditions [43], participants with shock encounters ruminate of recurrences [14]. Nevertheless, they were relieved that the device functioned and had no qualms over its necessity $[18,30]$. In contrast, participants without shock encounters ruminate possible future shocks $[14,17,18,20,42]$ and at times, they continued to doubt the device $[14,18,26]$. Similar to non-shock participants, those with phantom shocks also became less trusting of the ICD as they were alarmed and confused over their reactions to future shocks [37].

\section{Impacting self-identity, self-image and self-perception}

ICD implantation influenced one's body image perception $[17,20,38]$. Starrenburg et al. [35] found that females were associated with poorer device-related acceptance than males due to body image concerns. This is congruent with females' qualitative accounts of embarrassment associated with wearing clothes that reveal their scarring $[20,26]$. This may be due to greater societal expectation and emphasis on beauty in women compared to men. Moreover, according to Vazquez et al. [25], younger women tend to experience more image concerns than middle-aged and older women. Moreover, participants were conscious of the 
physical protrusion, arm movements, and lying down due to the awareness of the ICD in their chest $[17,42]$. Some participants, however, were dissatisfied with having foreign objects inserted as it made them feel being kept alive by machines [14].

Driving restrictions also resulted in poor self-identity and self-perception where participants reported feeling 'handicapped', uselessness, loss of dignity, and low selfrespect [12]. They viewed losing their driving license has depleted their overall well-being [30] as it is associated with the loss of independence, increasing reliance on others, and being limited in mobility and social life [12]. Nevertheless, the majority who drove before their ICD implantation had resumed driving after the restricted period [38]. Furthermore, if their license were revoked, it could have dire consequences on their employment and financial security $[12,18,20,30,42]$.

\section{Needing support and maintaining relationships}

Participants with adequate support, help, and empathy from their family and social networks had better recovery and adjustments [12, 15, 20, 30, 38, 43, 47]. During the period of driving restrictions, they were transported around [30] and prevented from engaging in certain activities that were deemed risky [38]. However, not everyone was comfortable to receive help [12]. Concerns were raised regarding overprotection $[15,23,26,38,43]$ as it made them feel dependent or being a burden $[15,18]$. While some had attempted to stop their family from constantly checking on them [47], others tolerated this positively [43, 47]. By comparison, most participants felt isolated as they had lost the most important person around the time of their diagnosis and were resistant to establish new connections for fear of loss [18]. Ironically, they also emphasised the importance of independence and self-reliance to preserve self-respect [18].

Participants who feared being alone or were reluctant to go out unaccompanied [30, 38, 44] experienced reduced social activity and became dependent [38]. Being protective could also strengthen couples' relationships [47]. Most of them became appreciative of their partners who were their pillars of support [43] and listeners in times of need [30]. However, there were also instances where reductions in sexual intimacy caused couples to become more emotionally distant $[42,43]$.

There was a general lack of professional support from the healthcare team $[26,30]$. Nurses were viewed as knowledge experts rather than listeners or patient advocates [18]. The lack of continuity in clinical care during follow-ups reduced patients' confidence [30] to receive support. Moreover, time constraint during follow-ups contributes to unmet emotional needs [30]. Participants also recalled encountering staff who were indifferent or uncomfortable with discussing sexual concerns [23].
Some participants accepted the uncertainty because they did not wish to bother or were unable to contact their healthcare professionals [26]. Several studies found that non-constructive support provided by healthcare professionals often led to more insecurity, psychological distress, and reduced device acceptance [30-32]. Nevertheless, there were also participants who reported receiving positive support from their healthcare team $[15,26,30]$. Such experiences varied between individuals due to potential subjectivity in how participants perceived the support based on their personal encounters.

In some studies, participants favoured joining and learning from support groups comprising of members with similar demographics and ICD experiences [14, 15, 44]. Specifically pertaining to sexual concerns, some had preferred to discuss with a support group member who is knowledgeable and experienced [23]. However, there were also others who, despite wanting to connect with ICD recipients, did not favour joining support groups [18] due to inconvenience, lack of anonymity, and on negative vibes $[15,18]$. Online support chat rooms could be an alternative for these participants $[15,20]$.

\section{Identifying learning needs}

Due to the short-term inpatient stay, limited information was obtained from healthcare professionals $[15,38]$. Moreover, participants were less receptive to the patient education provided in the stressful pre-ICD implantation period [12]. Although they were given resources for information [15], some still had queries [18] and were dissatisfied with the adequacy of the information provided $[15,26,30,38]$, particularly on driving restrictions and sexual concerns $[12,23]$. This could potentially be due to the lack of individualised advice and information reinforcements $[15,23]$. Some studies had also highlighted the lack of consistency in the information given by various healthcare professionals [12,38]. A qualitative study by Svanholm et al. [48] revealed that some of the octogenarians were unsure if they had suffered shocks throughout their lives because of misunderstandings on shocks. Evidently, incomplete patient education could result in participants' misinterpretations on their conditions.

A review of the articles identified 18 distinct learning needs which could be categorised into 4 main areas. These include: (1) general information on ICD where patient education on the functions, shocks, impacts, battery lifespan, and follow-ups pertaining to ICD should be given $[14,15,23,30,38]$; (2) diagnosis consisting of information on cardiac conditions, medications, and side effects of sudden cardiac deaths [14, 15, 18]; (3) living with ICD covers post-discharge advice on concerns like driving restrictions, resuming sexual activities, overcoming inconveniences, using electrical appliances and phones, appropriate physical activities, 
and swimming [12, 15, 18, 23, 38]; and (4) advanced planning for ICD deactivation [18].

\section{Developing coping strategies}

A cross-sectional study conducted in Sweden found that ICD recipients seldom use coping strategies and, among those used, optimism was most frequently used and highly effective [26]. Sometimes participants might have used coping mechanisms unknowingly as it occurred to them as their usual way of managing their everyday life and it had become a norm. Thus, it might not have occurred to them that these were actually also ways of coping with life after ICD implantation. Furthermore, at the moment where this study was conducted, most participants were already into their 6 to 24 months' post-implantation and might have already adapted to the device. Thus, they would report requiring less coping strategies. A future recommendation would be to explore the coping strategies used by the participants when faced with everyday crisis prior to the implantation and compare against post-implantation findings at several time intervals to find out the changes in coping strategies as well as isolating those that are specifically used for managing ICD issues.

Most qualitative studies did not explicitly state the participants' coping strategies and thus inference was made from their account. Several coping strategies were implicitly communicated with information belonging to subthemes like psychosocial distress or life adjustments and had to be extracted out. This review identified 12 main coping strategies which include: (1) optimistic interpretation of life situations [20, 26, 44, 47]; (2) talking about it and educating others [20]; (3) developing a strong willpower to live on [26, 44, 47]; (4) understanding own diagnosis to reduce uncertainty [14]; (5) re-evaluating outlook of life and prioritising goals [15, 20, 26, 43, 47]; (6) searching for meanings and rationalising situations [14, 37]; (7) religion and fatalism [15, 38, 43, 47]; (8) acceptance which could refer to either grateful acceptance or resigned acceptance [42]; (9) concealment of fears [42]; (10) distracting oneself with other activities and suppressing thoughts regarding diagnosis [26]; (11) evasiveness and avoidance [42-44, 47]; and lastly, (12) resignation $[42,43]$. The first nine coping strategies could be considered as either neutral or adaptive while the remaining three tend to be more maladaptive. Nevertheless, such determination is subjective and dependent on one's perception. Despite the variety of coping strategies identified, there was little information provided on its frequency and efficacy.

\section{Making adjustments and gaining acceptance}

Adaptations to limitations in life after ICD involves stages. In the initial period, it was about managing post- operative pain and negative emotions [15]. Most pain was experienced in the post-surgical stage and reduced thereafter [17]. Besides the surgical wound, pain was also experienced in the arm adjacent to the device due to restricted movements [42]. For the majority, such negative emotions usually dissipate after several weeks to months [20] as one learns to cope and eventually accept. Similarly, a longitudinal study by Carroll \& Hamilton [45] reported improvement in the mental health score on the Medical Outcomes Study Short Form-36 (SF-36) and reduced psychological distress score on the Profile of Mood States (POMS) by 6 months' post-implantation. Another longitudinal study by Verkerk et al. [36] also found that the mental health score on SF-36 was reduced at pre-implantation and 2 months but improved over time to be comparable with the population norm at 6 and 12 months. However, those with complications or shocks would require a longer adjustment time to build up their confidence [44].

Three qualitative findings had reported a reduction in physical functioning and activities in the initial period due to body weakness, discomfort, and reduced energy $[20,30]$. These findings were consistent with the quantitative study by Verkerk et al. [36] which also reported a reduction in physical functioning score on Short Form-36 (SF-36) health survey at 2 and 6 months but improved over time to be on par with the general population at 12 months. On the contrary, Conelius [17] reported that participants actually experienced more energy and better physical functioning post-implantation. A possible explanation implied from Conelius [17] was that these participants trusted the device to protect them and were therefore more daring to engage in physical activities compared to the pre-implantation stage where they could have been more ill. However, no information was provided regarding the severity of their cardiac diagnosis and baseline physical functioning level for comparison with other studies.

Generally, participants attempted to resume their normal routine $[15,17,20,22,23,26,44]$ and made adjustments to better control their lives. Some did so by placing personal restrictions and engaging in careful planning to balance activities with what was appropriate for their health [26]. Others began changing maladaptive habits to embrace a healthier lifestyle, reducing working hours to optimize life, and also avoiding activities that trigger shocks $[14,20,26,38]$. Instead of adhering to restrictions, some participants assessed their capabilities and risks as they successively tested their limits to increase physical level [14, 26, 44].

While older adults were concerned with self-care and functioning independently [47], younger adults were more concerned with developmental transitional tasks like family planning and childbearing [20]. Some were 
concerned about the heredity of the cardiac conditions and decided against childbearing while those with existing children that might require ICD in future had started preparing them for it [20]. Moreover, the participants approached sexual activities more carefully by altering patterns of sexual frequency and duration [23, 43].

\section{Planning for the end}

A study conducted by Pedersen et al. [34] on 294 participants found that $68 \%$ of them were aware of the option for ICD deactivation or battery replacement refusal during the end-of-life whereas a smaller-scaled study on 54 participants by Raphael et al. [49] yielded only $38 \%$. This difference could had been partially subjected to personal variations in the patient education provided by healthcare professionals. Moreover, the timing of discussing such issues also varied according to the practices of the settings where the studies were conducted as well as the patients' conditions, ICD implantation stage, and their readiness for enhanced information. Nevertheless, the poor understanding or the lack of knowledge in ICD deactivation in both studies revealed a lack of awareness regarding end-of-life planning. Similarly, qualitative findings also reported that most of the participants interviewed expressed unawareness of the option for ICD deactivation and that some even had the misconception of equating deactivation to euthanasia $[11,48]$. Furthermore, another study conducted by Stromberg et al. [29] on 3067 participants reported that only $3 \%$ had full scores on the Experiences, Attitudes, and Knowledge of End-of-Life Issues in ICD (EOL-ICD), with 29\% in the 25th percentile. Notably, these findings showed that more information regarding advance planning should be given.

Insufficient knowledge on end-of-life issues often cause greater indecisiveness or making decisions that might not attain a high quality of the end-of-life years $[11,29]$. Some participants had either requested for more information or expressed the willingness to be involved in such discussions with their physicians $[11,49,50]$ and most had preferred to know of the options prior to their implantation $[34,49]$. According to the findings in a study, the participants' favourable attitudes towards ICD deactivation was independently associated with the avoidance of shocks during dying as they wished for a worthy and natural death [34].

\section{Discussion}

This systematic review examined recent literature regarding the perceptions and experiences of patients living with ICD. The analysis of both quantitative and qualitative findings provided a deeper and richer insight into their quality of life, coping strategies adopted, as well as learning needs. However, caution should be exercised when interpreting these results due to the methodological limitations identified in most of the reviewed articles.

Firstly, some of the experiences recounted by the participants might inevitably be influenced by their underlying cardiac conditions, co-morbidities, and life stressors which also make up their life situations. As such, it would be difficult for participants to dissociate from other inter-related factors in their lives and share on experiences solely relating to ICD. In particular, the participants' psychological and emotional states, as well as life adjustments, could have been partly influenced by their newly-diagnosed cardiac conditions or life-threatening encounters that warrant the ICD implantation. Secondly, it was not clearly-stated in most studies whether the participants' ICD shock history were obtained from the medical records by researchers or participants' self-reports. Thus, this posed a challenge in determining if the shocks described during the qualitative interviews were phantom or truly objective experiences. Despite the lack of objective measurement, phantom shocks were described with such strong conviction that they possessed a similar physical reality as objective shocks. Just as phantom limb sensations were experienced by amputees, phantom shocks experienced by ICD recipients should not be disregarded. Moreover, researchers conducting future qualitative research on objective or phantom shocks should be blinded on the participants' shock experience so as to reduce the researchers' influence on the participants' account.

Ever since Kowey et al. [51] reported on the first incidence of phantom shock experience in 1992, there are still no studies in the present that has come up with a scientific account for phantom shocks. Bilanovic et al. [37] proposed a possible explanation that the participants might have perceived sub-threshold cardiac arrhythmias which fell short of being detected by the ICD as a shock therapy. This corroborated with the findings presented in another study by Kraaier et al. [52] where phantom shocks in the primary prevention group were related to a history of atrial fibrillation and potentially patients might have misinterpreted the symptoms of arrhythmia for phantom shocks. Likewise, the experiences and needs of ICD recipients with phantom shocks were also underexplored as evident by the fact that only one study published within the last 10 years was located during the systematic search. Although they belonged to a smaller subset of the ICD population, patients with phantom shocks would present different perceptions and needs. In this review, the comparison of experiences with phantom and objective shocks were limited due to the lack of published studies on phantom shocks. As such, future studies could look into exploring the perceptions of ICD recipients with phantom shock encounters. In addition, objective shocks could either be appropriate or inappropriate shock therapies delivered 
and since the MADIT-RIT study findings in 2012, changes to the ICD programming had reduced occurrences of inappropriate shock therapies [53]. Nevertheless, the differences in experiences among patients with appropriate and inappropriate shocks could be a potential area of future research interest. While the experiences of ICD recipients had been relatively well-explored in both quantitative and qualitative studies, and the majority of them were conducted in Western contexts. Only two of the studies were conducted in Asian settings [13, 14]. This revealed a lack of studies being conducted in Asian settings pertaining to this area where the cultural contexts can influence patients' experiences, coping, and needs differently despite having the same implantation. It is only by examining such differences that healthcare professionals can provide more relevant and culturally-sensitive care.

In addition, there is a greater number of studies focusing on the physical, psychosocial, and emotional impacts as compared to the other domains like spiritual, socioeconomic, sexual, self-identity, and childbearing concerns. As these domains tend to involve more sensitive and closeto-the-heart issues, most participants would not freely talk about them unless raised by the researchers. Even so, some participants might be uncomfortable sharing such information with someone they had not established any rapport with. This would be a challenge especially for qualitative studies taking on a phenomenological design where a grand tour question is being posed at the beginning and the participants control the direction of the conversation till they have nothing more to say. Furthermore, there were fewer studies examining the experiences related to more specific issues like ICD recalls, end-of-life ICD deactivation, battery replacement refusal, or phantom shocks. ICD recalls refers to cases of device malfunction that would require closer monitoring rather than explanation [54]. Although these instances are rare, such experiences could be distressful and more studies are warranted in this area as well. In the recent years, there are more studies conducted to explore the experiences of patients under telemonitoring or remote home monitoring which would had implications for future practices.

\section{Conclusion}

Although a careful systematic literature search was conducted, the search strategy may not have included all the relevant published literature. In addition, the differences in psychological impacts between appropriate and inappropriate ICD shocks may provide an interesting perspective. However, this is not included in this review as most of the articles included for this review did not differentiate between appropriate and inappropriate ICD shocks.

Nevertheless, this review indicates that ICD recipients experienced the transition from stages of uncertainty in the initial phase, to the adjustment phase, where they started to adapt and make life modifications, and finally attaining acceptance of self and trust in the ICD. It is a constant process of self-reflection, reorientation of their life perspectives, making sense of these changes, and moving on with life. Current evidence highlights the need to explore the perceptions and experiences of patients living with ICD in Asian settings.

As evident from the findings of this review, healthcare professionals tend to over-emphasise the scientific and clinical aspects rather than their patients' actual concerns such that the lack of constructive professional support was found to inflict greater psychological distress among ICD recipients. Unlike trained healthcare professionals, most patients, being laypersons, would not be able to understand the significance of clinical results and are therefore more concerned with their quality of life and normal functioning post-implantation. This misalignment in priorities could have attributed to the dissatisfaction among ICD recipients. In order to provide good targeted care for these patients, it is pertinent for healthcare professionals to acknowledge that patients are partners in care and they have the rights to partake in the management of their own health. By listening to their patients' concerns and daily lives, healthcare professionals could obtain a better understanding of their coping and establish therapeutic alliance to assist patients in further improving their quality of life.

\section{Abbreviations \\ ICD: Implantable cardioverter defibrillator; OS: Objective shock; PS: Phantom shock; PTSD: Post-traumatic stress disorder; QOL: Quality of life}

\section{Acknowledgements \\ The authors would like to acknowledge the Ministry of Education, Academic Research Fund - Tier 1 for the support to conduct this study, and would also like to extend their appreciation and acknowledgements to the study team members for their time and effort spend in this study.}

\section{Funding}

The study was funded by the Ministry of Education, Academic Research Fund - Tier 1, Singapore (Award number: T1-2013 APR -05).

\section{Availability of data and materials}

The datasets used for this review are available from the corresponding author.

\begin{abstract}
Authors' contributions
OSL conducted the literature search, analysed the data analysis, and drafted the manuscript. HGH analysed the data and critically reviewed and revised the manuscript. YD critically reviewed and revised the manuscript. WW designed the study, analysed the data, and drafted the manuscript. All authors read and approved the final manuscript as submitted.
\end{abstract}

\section{Competing interests}

The authors declare that they have no competing interests.

Consent for publication

Not applicable.

Ethics approval and consent to participate Not applicable. 


\section{Author details}

Alice Lee Centre for Nursing Studies, Yong Loo Lin School of Medicine, National University of Singapore, Level 2, Clinical Research Centre, Block MD 11,10 Medical Drive, Singapore, Singapore. ${ }^{2}$ Department of Pharmacology, Yong Loo Lin School of Medicine, National University of Singapore, Singapore, Singapore.

Received: 7 April 2016 Accepted: 9 November 2016

\section{Published online: 17 November 2016}

\section{References}

1. Eisenberg MS. Resuscitate: how your community can improve survival from sudden cardiac arrest. 2nd ed. Seattle: University of Washington Press; 2013.

2. Mehra R. Global public health problem of sudden cardiac death. J Electrocardiol. 2007;40(6):S118-22

3. Israel CW. Mechanisms of sudden cardiac death. Internal Heart J. 2014;66 Suppl 1:S10-7.

4. Chugh SS, Reinier K, Teodorescu C, Evanado A, Kehr E, Samara MA, et al. Epidemiology of sudden cardiac death: clinical and research implications. Prog Cardiovasc Dis. 2008:51(3):213-28.

5. Hayes DL, Asirvatham SJ. Dictionary of cardiac pacing, defibrillation, resynchronization, and arrhythmias. Rochester: Mayo Foundation for Medical Education and Research; 2007.

6. Knight BP. Patient information: Implantable cardioverter-defibrillators (Beyond the basics) 2014. Retrieved from http://www.uptodate.com/contents/implantablecardioverter-defibrillators-beyond-the-basics (accesed 10 Jan 2016).

7. Hlatky MA, Sanders GD, Owens DK. Evidence-based medicine and policy: the case of the implantable cardioverter defibrillator. Health Affair. 2005;24(1):42-51.

8. British Medical Association Library. BMA library - Medline plus: Basic course notes for ovidSP 2012 [Booklet]. Retrieved from file:///C:/Users/Maybelline/ Downloads/Medline\%20Plus\%20basic\%20course\%20manual\%202012.pdf (accessed on 10 Jan 2016).

9. The Joanna Briggs Institute. Edition 2014 reviewers' manual [Booklet]. Australia: The Joanna Briggs Institute; 2014.

10. Braun V, Clarke V. Using thematic analysis in psychology. Qual Res Psychol. 2006;3(2):77-101.

11. Fluur C, Bolse K, Strömberg A, Thylén I. Patients' experiences of the implantable cardioverter defibrillator (ICD); with a focus on battery replacement and endof-life issues. Heart Lung. 2013;42(3):202-7.

12. Johansson I, Strömberg A. Experiences of driving and driving restrictions in recipients with an implantable cardioverter defibrillator-the patient perspective. J Cardiovasc Nurs. 2010;25(6):E1-E10.

13. Chair SY, Lee CK, Choi KC, Sears SF. Quality of life outcomes in Chinese patients with implantable cardioverter defibrillators. Pacing Clin Electrophysiol. 2011;34(7):858-67.

14. Saito N, Taru C, Miyawaki I. Illness experience: living with arrhythmia and implantable. Kobe J Med Sci. 2012;58(3):E72-81.

15. Bolse K, Hamilton G, Flanagan Caroll DL, Fridlund B. Ways of experiencing the life situation among United States patients with an implantable cardioverter defibrillator: a qualitative study. Prog Cardiovasc Nurs. 2005;20(1):4-10.

16. Carroll DL, Hamilton GA. Quality of life in implanted cardioverter defibrillator recipients: the impact of a device shock. Heart Lung. 2005;34(3):169-78.

17. Conelius J. A woman's experience: living with an implantable cardioverter defibrillator. Appl Nurs Res. 2015;28(2):192-6.

18. Flanagan JM, Carroll DL, Hamilton GA. The long-term lived experience of patients with implantable cardioverter defibrillators. Med Surg Nurs. 2010;19(2):113.

19. Groeneveld PW, Matta MA, Suh JJ, Yang F, Shea JA. Quality of life among Implantable Cardioverter Defibrillator recipients in the primary prevention therapeutic era. Pacing Clin Electrophysiol. 2007;30(4):463-71.

20. McDonough A. The experiences and concerns of young adults (18-40 years) living with an implanted cardioverter defibrillator (ICD). Eur I Cardiovasc Nurs. 2009;8(4):274-80

21. Myers GM, James GD. Social support, anxiety, and support group participation in patients with an implantable cardioverter defibrillator. Prog Cardiovasc Nurs. 2008;23(4):160-7.

22. Salmoirago-Blotcher E, Crawford S, Tran C, Goldberg R, Rosenthal L, Ockene I. Spiritual well-being may buffer psychological distress in patients with implantable cardioverter defibrillators. J Evid Based Complement Altern Med. 2012;17(3):148-54.
23. Steinke EE, Gill-Hopple K, Valdez D, Wooster M. Sexual concerns and educational needs after an implantable cardioverter defibrillator. Heart Lung. 2005;34(5):299-308.

24. Thomas SA, Friedmann E, Gottlieb SS, Liu F, Morton PG, Chapa DW, et al. Changes in psychosocial distress in outpatients with heart failure with implantable cardioverter defibrillators. Heart Lung. 2009;38(2):109-20.

25. Vazquez LD, Kuhl EA, Shea JB, Kirkness A, Lemon J, Whalley D, et al. Agespecific differences in women with Implantable Cardioverter Defibrillators: an international multi center study. Pacing Clin Electrophysiol. 2008; 31(12):1528-34.

26. Flemme I, Hallberg U, Johansson I, Strömberg A. Uncertainty is a major concern for patients with implantable cardioverter defibrillators. Heart Lung. 2011:40(5):420-8.

27. Flemme I, Johansson I, Strömberg A. Living with life-saving technologycoping strategies in implantable cardioverter defibrillators recipients. J Clin Nurs. 2012;21(3-4):311-21.

28. Flemme I, Edvardsson N, Hinic H, Jinhage BM, Dalman M, Fridlund B. Longterm quality of life and uncertainty in patients living with an implantable cardioverter defibrillator. Heart Lung. 2005;34(6):386-92.

29. Strömberg A, Fluur C, Miller J, Chung ML, Moser DK, Thylen I. ICD Recipients' understanding of ethical issues, ICD function, and practical consequences of withdrawing the ICD in the end-of-life. Pacing Clin Electrophysiol. 2014; 37(7):834-42.

30. Morken IM, Severinsson E, Karlsen B. Reconstructing unpredictability: experiences of living with an implantable cardioverter defibrillator over time. J Clin Nurs. 2010;19(3-4):537-46.

31. Morken IM, Norekvål TM, Bru E, Larsen Al, Karlsen B. Perceptions of healthcare professionals' support, shock anxiety and device acceptance among implantable cardioverter defibrillator recipients. J Adv Nurs. 2014;70(9):2061-71.

32. Morken IM, Bru E, Norekvål TM, Larsen Al, Idsoe T, Karlsen B. Perceived support from healthcare professionals, shock anxiety and post-traumatic stress in implantable cardioverter defibrillator recipients. J Clin Nurs. 2014;23(3-4):450-60.

33. Habibović $M$, van den Broek KC, Theuns DA, Jordaens $L$, Alings $M$, van der Voort PH. Gender disparities in anxiety and quality of life in patients with an implantable cardioverter-defibrillator. Europace. 2011;13(12):1723-30.

34. Pedersen SS, Chaitsing R, Szili-Torok T, Jordaens L, Theuns DA. Patients' perspective on deactivation of the implantable cardioverter-defibrillator near the end of life. Am J Cardiol. 2013;111(10):1443-7.

35. Starrenburg A, Pedersen S, den Broek K, Kraaier K, Scholten M, Palen J. Gender differences in psychological distress and quality of life in patients with an ICD 1-Year postimplant. Pacing Clin Electrophysiol. 2014;37(7):843-52.

36. Verkerk AJ, Vermeer AM, Smets EM, Dekker LR, Wilde AA, Van Langen IM. Quality of life in young adult patients with a cardiogenetic condition receiving an ICD for primary prevention of sudden cardiac death. Pacing Clin Electrophysiol. 2015;38(7):870-7.

37. Bilanovic A, Irvine J, Kovacs AH, Hill A, Cameron D, Katz J. Uncovering phantom shocks in cardiac patients with an implantable cardioverter defibrillator. Pacing Clin Electrophysiol. 2013;36(6):673-83.

38. Mert $\mathrm{H}$, Argon $\mathrm{G}$, Aslan $\mathrm{O}$. Experiences of patients with implantable cardioverter defibrillator in Turkey: a qualitative study. IJCS. 2012;5(1):50-5.

39. Spindler $\mathrm{H}$, Johansen JB, Andersen K, Mortensen P, Pedersen SS. Gender differences in anxiety and concerns about the cardioverter defibrillator. Pacing Clin Electrophysiol. 2009;32(5):614-21.

40. Versteeg H, Baumert J, Kolb C, Pedersen SS, Denollet J, Ronel J. Somatosensory amplification mediates sex differences in psychological distress among cardioverter-defibrillator patients. Health Psychol. 2010;29(5):477.

41. Colloca L, Benedetti F. Nocebo hyperalgesia: how anxiety is turned into pain. Curr Opin Anaesthesiol. 2007;20(5):435-9.

42. Humphreys NK, Lowe R, Rance J, Bennett PD. Living with an implantable cardioverter defibrillator: the patients' experience. Heart Lung. 2016;45(1):34-40.

43. Palacios-Ceña D, Losa ME, Salvadores-Fuentes P, Alonso-Blanco C, Fernándezde-las-Peñas $C$. Experience of elderly Spanish men with an implantable cardioverter-defibrillator. Geriatr Gerontol Int. 2011;11(3):320-7.

44. Williams AM, Young J, Nikoletti S, McRae S. Getting on with life: accepting the permanency of an implantable cardioverter defibrillator. Int J Nurs Pract. 2007;13(3):166-72.

45. Carroll DL, Hamilton GA. Long-term effects of implanted cardioverterdefibrillators on health status, quality of life, and psychological state. Am J Crit Care. 2008;17(3):222-30.

46. Jacq F, Foulldrin G, Savouré A, Anselme F, Baquelin-Pinaud A, Cribier A. A comparison of anxiety, depression and quality of life between device shock 
and nonshock groups in implantable cardioverter defibrillator recipients. Gen Hosp Psychiat. 2009;31(3):266-73.

47. Palacios-Ceña D, Losa ME, Fernández-de-las-Peñas C, Salvadores-Fuentes $P$. Living with life insurance: a qualitative analysis of the experience of male implantable defibrillator recipients in Spain. J Clin Nurs. 2011;20(13-14):2003-13.

48. Svanholm JR, Nielsen JC, Mortensen P, Christensen CF, Birkelund R. Refusing Implantable Cardioverter Defibrillator (ICD) peplacement in elderly persons-the same as giving up life: a qualitative study. Pacing Clin Electrophysiol. 2015;38(11):1275-86.

49. Raphael CE, Wing KM, Stain N, Wright I, Francis DP, Kanagaratnam P. Implantable cardioverter defibrillator recipient attitudes towards device deactivation: how much do patients want to know? Pacing Clin Electrophysiol. 2011;34(12):1628-33.

50. Herman D, Stros P, Curila K, Kebza V, Osmancik P. Deactivation of implantable cardioverter-defibrillators: results of patient surveys. Europace. 2013;15(7):963-9.

51. Kowey PR, Marinchak RA, Rials SJ. Things that go bang in the night. N Engl J Med. 1992;327(26):1884

52. Kraaier K, Starrenburg A, Verheggen R, Van der Palen J, Scholten M. Incidence and predictors of phantom shocks in implantable cardioverter defibrillator recipients. Neth Heart J. 2013;21(4):191-5.

53. Moss AJ, Schuger C, Beck CA, Brown MW, Cannom DS, Daubert JP, et al. Reduction in inappropriate therapy and mortality through ICD programming. N Engl J Med. 2013;367(24):2275-83.

54. Kirian KB, Sears SF, Shea JB. How to respond to an implantable cardioverterdefibrillator recall. Circulation. 2009;119(5):e189-91.

\section{Submit your next manuscript to BioMed Central and we will help you at every step:}

- We accept pre-submission inquiries

- Our selector tool helps you to find the most relevant journal

- We provide round the clock customer support

- Convenient online submission

- Thorough peer review

- Inclusion in PubMed and all major indexing services

- Maximum visibility for your research

Submit your manuscript at www.biomedcentral.com/submit

C) Biomed Central 